\title{
The Brunt-Väisälä frequency of rotating tokamak plasmas
}

\author{
J.W. Haverkort ${ }^{\mathrm{a}, \mathrm{b}, *}$, H.J. de Blank ${ }^{\mathrm{a}}$, B. Koren ${ }^{\mathrm{b}, \mathrm{c}}$

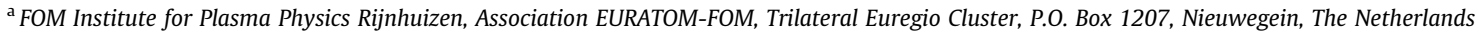 \\ ${ }^{\mathrm{b}}$ Centrum Wiskunde \& Informatica (CWI), P.O. Box 94079, Amsterdam, The Netherlands \\ ${ }^{\mathrm{c}}$ Mathematical Institute, Leiden University, P.O. Box 9512, 2300 RA Leiden, The Netherlands
}

\section{A R T I C L E I N F O}

\section{Article history:}

Available online 12 March 2011

\section{Keywords:}

Ideal magnetohydrodynamics

Plasma flow

Toroidal rotation

Grad-Shafranov equation

Analytical solutions

Convective effect

Continuous spectrum

\begin{abstract}
A B S T R A C T
The continuous spectrum of analytical toroidally rotating magnetically confined plasma equilibria is investigated analytically and numerically. In the presence of purely toroidal flow, the ideal magnetohydrodynamic equations leave the freedom to specify which thermodynamic quantity is constant on the magnetic surfaces. Introducing a general parametrization of this quantity, analytical equilibrium solutions are derived that still posses this freedom. These equilibria and their spectral properties are shown to be ideally suited for testing numerical equilibrium and stability codes including toroidal rotation. Analytical expressions are derived for the low-frequency continuous Alfvén spectrum. These expressions still allow one to choose which quantity is constant on the magnetic surfaces of the equilibrium, thereby generalizing previous results. The centrifugal convective effect is shown to modify the lowest Alfvén continuum branch to a buoyancy frequency, or Brunt-Väisälä frequency. A comparison with numerical results for the case that the specific entropy, the temperature, or the density is constant on the magnetic surfaces yields excellent agreement, showing the usefulness of the derived expressions for the validation of numerical codes.
\end{abstract}

(c) 2011 Elsevier Inc. All rights reserved.

\section{Introduction}

One of the very few viable ways to confine a plasma at the high temperatures required for nuclear fusion, is using magnetic fields. One of the most promising designs suitable for the commercial production of energy is the tokamak, in which the plasma is confined in the shape of a torus. A tokamak force equilibrium can be described quite well by a balance between the pressure gradient, the Lorentz force, and centrifugal forces. This force balance can be described by the fluid model of magnetohydrodynamics (MHD). Assuming axisymmetry, the static MHD equations can be reduced to a single partial differential equation, called the Grad-Shafranov equation [1,2]. The solution $\psi$ is a stream function for the poloidal magnetic field, and can be used as a coordinate that labels the magnetic surfaces.

Given the dependence of the pressure and the toroidal magnetic field on $\psi$, the solution of the Grad-Shafranov equation gives $\psi$ as a function of the spatial coordinates, completing the description of the equilibrium. Assuming this dependence to be linear, a simple polynomial solution was obtained by Solov'ev [3]. Incidentally, this solution is identical to Hill's solution for the Stokes stream function of a spherical vortex [4], which long preceded the conception of the idea of a tokamak in the 1950's. Solutions expressed in special mathematical functions were also found for a quadratic dependence [5-7] and a mixed linear and quadratic dependence [8-11]. The most general linear solution is obtained in Refs. [12,13]. More exotic dependencies yielding analytical solutions are considered in Refs. [14,15]. Convenient homogeneous solutions are provided in Refs. [16,17].

\footnotetext{
* Corresponding author at: Centrum Wiskunde \& Informatica (CWI), P.O. Box 94079, Amsterdam, The Netherlands.

E-mail address: J.W.Haverkort@cwi.nl (J.W. Haverkort).
} 
In the presence of toroidal rotation, one additionally has to specify how the angular rotation frequency depends on $\psi$. Assuming that the ratio between the angular frequency squared and the static temperature is constant and that the magnetic surfaces have constant temperature or entropy, analytical solutions were found for a linear [18] or a mixed linear and quadratic dependence [19] of the pressure and the toroidal magnetic field on $\psi$. For isothermal magnetic surfaces, solutions for a quadratic or mixed dependence were already obtained in Refs. [20,21], or in spherical coordinates in Ref. [22]. For more general flows, solutions have been found assuming plasma incompressibility, see e.g. Refs. [23,24].

All existing analytical equilibrium solutions including toroidal flow, make an assumption on which quantity is constant on the magnetic surfaces. In the present work, we assume a general parametrization of this quantity from the outset. The freedom of this choice is retained in the equilibrium equation and the subsequently derived class of analytical solutions. These analytical solutions are generalizations of those of Ref. [18] and without rotation reduce to the polynomial Solov'ev solution [3]. For numerical stability calculations, an accurate numerical representation of the equilibrium is required. In order to test the accuracy of the equilibrium solver, the Solov'ev solution is frequently used [25-29].

Various equilibrium codes that include toroidal rotation exist. Most of these codes assume isothermal magnetic surfaces [30-36] while some assume flux surfaces of constant density [37,38]. The equilibrium code FINESSE [39] allows in case of purely toroidal flow the freedom to choose either the temperature, the density, or the entropy to be constant within the magnetic surfaces [40]. Only rarely, analytical solutions have been used to test an equilibrium code including toroidal flow [35]. In the present work we will use the derived analytical solutions, to test the convergence behavior of FINESSE.

The Solov'ev equilibrium solution [3] is also frequently used for stability calculations as a standardized reference equilibrium [27,28,41-43]. For stability calculations including toroidal flow, less well-defined reference test cases are generally used to benchmark numerical codes. Again, the derived analytical solutions would be ideally suited to serve as a standardized reference equilibrium including rotation.

Also in the analytical stability calculations in this work, the freedom to specify which quantity is constant on the magnetic surfaces of the equilibrium is retained. An analytical expression is derived for the low-frequency continuous Alfvén spectrum of a large aspect ratio tokamak plasma, including the effects of compressibility and toroidal rotation. This expression still allows for the choice of which quantity is constant on the magnetic surfaces of the equilibrium, and as such generalizes the results of Refs. [44-47] for isothermal flux surfaces and the recent result of Ref. [48] for magnetic surfaces of constant density.

The lowest-frequency branch of the derived expression is shown to be equal to the Brunt-Väisälä-frequency associated with the centrifugal convective effect. For a tokamak plasma with magnetic surfaces of constant density, the convective instability destabilizes the continuous spectrum. Simulations show how instability spreads from a localized part of the tokamak to almost the entire plasma for sonic rotational velocities. For a tokamak with isothermal magnetic surfaces, rotation induces a gap in the continuous spectrum. Good correspondence between the continuum frequencies from numerical simulations and the derived analytical expression is obtained, showing its usefulness for the validation of stability codes.

\section{Magnetohydrodynamic equilibria}

In this section we will introduce the considered geometry and basic equations that are used in the analysis of the subsequent sections. Although this work pertains to toroidal rotation, in order to provide some context and introduce concepts that will arise in the stability analyses, Section 2.3 is devoted to more general equilibrium flows.

\subsection{Equations}

We start from the ideal magnetohydrodynamic (MHD) equations. These consist of the conservation equations for momentum, mass, and energy

$$
\begin{aligned}
& \rho \frac{\partial \mathbf{u}}{\partial t}+\rho \mathbf{u} \cdot \nabla \mathbf{u}+\nabla p-\mathbf{J} \times \mathbf{B}=0, \\
& \frac{\partial \rho}{\partial t}+\nabla \cdot(\rho \mathbf{u})=0, \\
& \frac{\partial S}{\partial t}+\mathbf{u} \cdot \nabla S=0
\end{aligned}
$$

together with the reduced Maxwell equations

$$
\begin{aligned}
& \nabla \times \mathbf{E}=-\partial \mathbf{B} / \partial t, \\
& \nabla \times \mathbf{B}=\mathbf{J}, \\
& \nabla \cdot \mathbf{B}=0
\end{aligned}
$$


and Ohm's law for ideally conducting moving media

$$
\mathbf{E}=-\mathbf{u} \times \mathbf{B},
$$

for the velocity $\mathbf{u}$, magnetic field $\mathbf{B}$, current density $\mathbf{J}$, pressure $p$, density $\rho$. The quantity $S \equiv p \rho^{-\gamma}$, with $\gamma$ the adiabatic constant, is related to the specific entropy. The electric field $\mathbf{E}$ is actually an auxiliary quantity that can be left out of the formulation by replacing Eqs. (1d) and (1g) by $\nabla \times(\mathbf{u} \times \mathbf{B})=\partial \mathbf{B} / \partial t$. In ideal MHD the same holds for $\mathbf{J}$, which can be left out of the formulation by inserting Eq. (1e) for $\mathbf{J}$ into Eq. (1a) to give

$$
\begin{aligned}
& \rho \frac{\partial \mathbf{u}}{\partial t}+\rho \mathbf{u} \cdot \nabla \mathbf{u}+\nabla p-(\nabla \times \mathbf{B}) \times \mathbf{B}=0 \\
& \frac{\partial \rho}{\partial t}+\nabla \cdot(\rho \mathbf{u})=0 \\
& \frac{\partial S}{\partial t}+\mathbf{u} \cdot \nabla S=0 \\
& \frac{\partial \mathbf{B}}{\partial t}-\nabla \times(\mathbf{u} \times \mathbf{B})=0 \\
& \nabla \cdot \mathbf{B}=0 .
\end{aligned}
$$

The stationary ideal MHD equations then follow using $\partial / \partial t=0$. Note that the vacuum magnetic permeability $\mu_{0}$ does not appear explicitly, because it can be scaled out of the equations by a proper dimensionalization of the physical variables. The energy Eq. (2c) is often referred to as the adiabatic equation of state, since it states that the quantity $S \equiv p \rho^{-\gamma}$, which is monotonic function of the specific entropy, is constant along the flow.

\subsection{Toroidal flow}

\subsubsection{Extended Grad-Shafranov equation}

In the following we will use the coordinate system $(R, Z, \phi)$ shown in Fig. 1, commonly used for toroidal problems. This coordinate system differs from the usual cylindrical coordinate system in the direction of $\phi$. We will assume axisymmetry around the $Z$-axis, making the toroidal angle $\phi$ an ignorable coordinate. A hat will be used to denote a unit vector. When the flow is purely toroidal $\mathbf{u}=R \Omega \hat{\boldsymbol{\phi}}$, the non-linear term in Eq. (2a) represents the centrifugal force density

$$
-\rho \mathbf{u} \cdot \nabla \mathbf{u}=\rho \Omega^{2} \mathbf{R}
$$

where $\mathbf{R}=R \nabla R$. For purely toroidal flow, i.e. without flow in the poloidal plane, the stationary versions of the continuity Eq. (2b) and energy Eq. (2c) are trivially satisfied due to axisymmetry. In order to further reduce the resulting equations, we split the magnetic field in a toroidal and poloidal part as follows:

$$
\begin{aligned}
& \mathbf{B}=\nabla \phi \times \nabla \psi+F \nabla \phi, \\
& \mathbf{J}=\nabla F \times \nabla \phi+\Delta^{*} \psi \nabla \phi,
\end{aligned}
$$

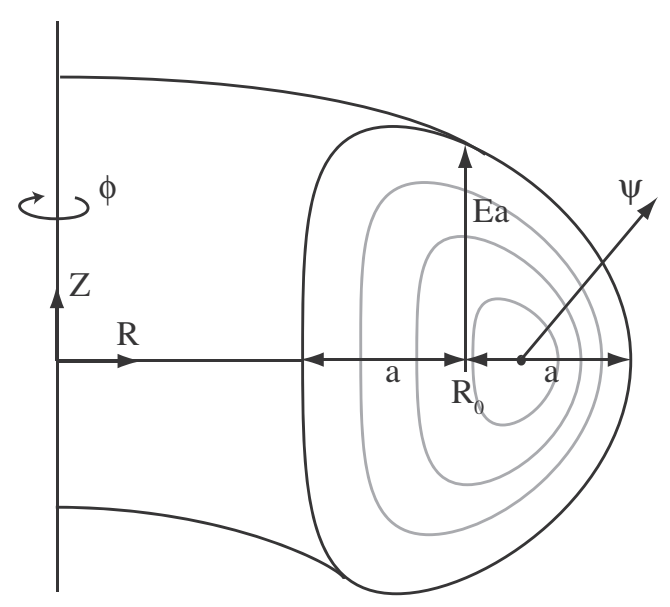

Fig. 1. A schematic overview of the tokamak geometry including the major radius $R_{0}$ and minor radius $a=\epsilon R_{0}$, giving an inverse aspect ratio $\epsilon \equiv a / R_{0}$. In the used coordinate system $(R, Z, \phi)$, the direction of $\phi$ differs from that of the cylindrical coordinate system $(R, \phi, Z)$. Note that the ellipticity $E$ differs from the commonly used definition. Also note that the magnetic axis, where the poloidal magnetic flux function $\psi$ has a minimum, differs from the geometrical axis at $R=R_{0}$ by the Shafranov shift. 
where $\nabla \phi=\hat{\boldsymbol{\phi}} / R$. Here $\psi$ and $F \equiv R B_{\phi}$ are stream functions of the poloidal part of $\mathbf{B}$ and $\mathbf{J}$ respectively. Because $\mathbf{B} \cdot \nabla \psi=0$, the magnetic field lies within surfaces of constant $\psi$, which can therefore be used to label the magnetic surfaces as indicated in Fig. 1. The quantity $\psi$ can be seen to be proportional to the poloidal magnetic flux, by integrating Eq. (4a) over an axisymmetric strip $S$ of width $\Delta \psi$. Using $\nabla \phi \times \nabla \psi=-\nabla \times(\psi \nabla \phi)$ and Green's theorem we obtain for the poloidal magnetic flux

$$
\int_{S} \mathbf{B} \cdot d \mathbf{S}=\int_{S} \nabla \phi \times \nabla \psi \cdot d \mathbf{S}=-\oint_{\partial S} \psi \nabla \phi \cdot d \mathbf{l}=2 \pi \Delta \psi .
$$

The toroidal current $J_{\phi}=\Delta^{*} \psi / R$ in Eq. (4b) is expressed in terms of the elliptic operator defined by

$$
\Delta^{*} \psi \equiv R^{2} \nabla \cdot\left(R^{-2} \nabla \psi\right)=R \frac{\partial}{\partial R}\left(\frac{1}{R} \frac{\partial \psi}{\partial R}\right)+\frac{\partial^{2} \psi}{\partial Z^{2}},
$$

to ensure that Ampere's law, Eq. (1e), is satisfied. Using again $\nabla \phi \times \nabla \psi=-\nabla \times(\psi \nabla \phi)$ and axisymmetry, Eq. (4a) for B manifestly satisfies Eq. (2e), $\nabla \cdot \mathbf{B}=0$. Eq. (1g), together with Eq. (4a), yields

$$
\mathbf{E}=\Omega \nabla \psi
$$

so that $\nabla \times \mathbf{E}=0$ is automatically satisfied when $\Omega$ is a function of $\psi$ only. Quantities like $\Omega$ that depend only on the poloidal flux $\psi$ are often called flux functions, or surface functions.

Within the above formulation, the only equilibrium equation that is not yet automatically satisfied is the momentum equation. A toroidal projection of Eq. (2a) with $\partial / \partial t=0$ leads to the observation that the Lorentz force $\mathbf{J} \times \mathbf{B}$ has no toroidal component. Inserting the expressions of Eqs. (4a) and (4b) yields $(\mathbf{J} \times \mathbf{B})_{\phi}=\nabla \psi \cdot(\nabla F \times \nabla \phi)=0$, so that $F=F(\psi)$ is a flux function. A second projection of the momentum equation, parallel to the poloidal magnetic field, yields

$$
\left.\frac{\partial p}{\partial R}\right|_{\psi}=\rho R \Omega^{2}
$$

showing that, within the magnetic surfaces of constant $\psi$, the centrifugal force will be balanced exclusively by pressure gradients. A final projection of Eq. (2a) in the direction of $\nabla \psi$ gives the extended Grad-Shafranov equation [18]

$$
\Delta^{*} \psi=-\frac{d \frac{1}{2} F^{2}}{d \psi}-\left.R^{2} \frac{\partial p}{\partial \psi}\right|_{R}
$$

which is the final equation for ideal axisymmetric equilibria with purely toroidal flow. Provided expressions for $F$ and $p$ are given, this elliptic Eq. (9) can be solved either numerically or analytically for $\psi$. The difference between Eq. (9) and the ordinary Grad-Shafranov equation for static equilibria is that the last term contains a partial derivative, because the pressure has to satisfy the additional Eq. (8). For static equilibria, the momentum equation $\nabla p=\mathbf{J} \times \mathbf{B}$ ensures $\mathbf{B} \cdot \nabla p=0$ so that $p=p(\psi)$.

\subsubsection{Solving for the pressure analytically}

In order to solve Eq. (9) one needs an expression for the pressure, satisfying Eq. (8). We will introduce the following general parametrization that satisfies this equation

$$
\begin{aligned}
& p=p_{s}(\psi)\left(1+R^{2} \Lambda(\psi) / \zeta(\psi)\right)^{\zeta(\psi)}, \\
& \rho=\rho_{s}(\psi)\left(1+R^{2} \Lambda(\psi) / \zeta(\psi)\right)^{\zeta(\psi)-1},
\end{aligned}
$$

where $\Lambda(\psi) \equiv \rho_{s} \Omega^{2} / 2 p_{s}, \zeta(\psi)$ is an arbitrary non-zero flux function, and the subscripts $s$ denote static quantities.

One has the freedom of specifying an arbitrary equation of state, for which we choose the ideal gas law

$$
p=\rho T,
$$

where a constant of proportionality is absorbed in the definition of the temperature $T$. The temperature can now be written as

$$
T=T_{s}(\psi)\left(1+R^{2} \Lambda(\psi) / \zeta(\psi)\right)
$$

where $T_{s}(\psi)=p_{s} / \rho_{s}$, so that $\Lambda(\psi)=\Omega^{2} / 2 T_{s}$.

Note from Eqs. (10) and (11) that, with $\gamma_{\zeta} \equiv \zeta /(\zeta-1)$, the following quantity is a flux function

$$
A(\psi) \equiv p \rho^{-\gamma_{\zeta}}=p_{s} \rho_{s}^{-\gamma_{\zeta}}
$$

For different $\zeta$, or equivalently different $\gamma_{\zeta}$, various physical variables can be assumed to be constant on the magnetic surfaces. In particular 


$$
A(\psi)=\left\{\begin{array}{lll}
\rho(\psi) \text { for } \zeta \rightarrow 1 & \left(\gamma_{\zeta} \rightarrow \infty\right), \\
T(\psi) \text { for } \zeta \rightarrow \infty & \left(\gamma_{\zeta} \rightarrow 1\right), \\
S(\psi) & \text { for } \zeta=\gamma /(\gamma-1) & \left(\gamma_{\zeta}=\gamma\right),
\end{array}\right.
$$

In the case of purely toroidal flow we thus find that in addition to $p_{s}(\psi), F(\psi)$, and $\Omega(\psi)$, there is a fourth flux function $A(\psi)$, or alternatively $\zeta(\psi)$ or $\gamma_{\zeta}(\psi)$, that directly influences the equilibrium solution $\psi(R, Z)$. Note that in the limit $\zeta \rightarrow \infty$, the factor $\left(1+R^{2} \Lambda(\psi) / \zeta\right)^{\zeta}$ becomes $\exp \Lambda R^{2}$. Eq. (10) for the pressure can now be inserted into the extended Grad-Shafranov Eq. (9) which can then be solved separately.

\subsection{General equilibrium flow}

Even though this paper deals with toroidal equilibrium flow, selected aspects associated with poloidal flow are considered in this section. For general equilibrium flows one has to solve an algebraic (Bernoulli) equation for the density and a generalized Grad-Shafranov equation simultaneously [49]. Here, we will only discuss the effect of poloidal flow on the freedom to choose $\zeta(\psi)$ arbitrarily. In section 2.3 .2 we consider an aspect of motion along the magnetic field lines that will play a role in later sections.

\subsubsection{Poloidal flow}

From Eq. (2d) it follows with $\partial / \partial t=0$ that $\mathbf{u} \cdot \nabla \psi=0$, implying that equilibrium plasma flow will always be within the magnetic surfaces only $[50,49]$. Apart from the toroidal rotation considered in this paper, plasma can also rotate poloidally. The stationary versions of Eqs. (2d) and (2b) are still satisfied when a flow $\Phi(\psi) \mathbf{B} / \rho$ is added to $\mathbf{u}$ so that

$$
\mathbf{u}=\frac{\Phi(\psi)}{\rho} \mathbf{B}+R \Omega(\psi) \hat{\boldsymbol{\phi}}
$$

Note that for non-zero $\Phi$, Eq. (2c) requires that $\mathbf{B} \cdot \nabla S=0$ so that the entropy is a flux function. This implies that $\gamma_{\zeta}$ in Eq. (14) can no longer be chosen arbitrarily, but assumes the value $\gamma_{\zeta}=\gamma$.

When heat conduction parallel to the field lines is faster than the plasma rotates poloidally, parallel heat exchange may ensure temperature equilibration of the magnetic surfaces. For small poloidal flow velocities, the stationary adiabatic energy equation $\mathbf{u} \cdot \nabla S$ may therefore be replaced by an isothermal equation $\mathbf{u} \cdot \nabla T$. For the description of faster phenomena like waves and instabilities, the adiabatic equation is often more appropriate. We note that the use of these two different energy equations is equivalent to using the same energy equation, with different adiabatic constants. This is nicely facilitated by the introduction of the 'adiabatic constant' $\gamma_{\zeta}$ of the equilibrium, separate from the adiabatic constant $\gamma$.

Whether the flux surfaces in a tokamak will tend to be isentropic so that $\gamma_{\zeta}=\gamma$, isothermal so that $\gamma_{\zeta} \rightarrow 1$, or something else, will therefore depend on the strength of the mechanism that drives poloidal rotation compared to the magnitude of the parallel heat conductivity. It has even been argued [51] that an assumption of incompressible equilibrium flow may be justified for tokamak experiments. Incompressibility entails that $\nabla \cdot \mathbf{u}=0$ so that $\rho$ is constant on magnetic surfaces and $\gamma_{\zeta} \rightarrow \infty$. The most appropriate value for $\gamma_{\zeta}$ may depend on the specific situation under consideration and $\gamma_{\zeta}$ may even assume different values on different magnetic surfaces. Not unlike the way $p_{s}(\psi)$ and $F(\psi)$ can be chosen to model experimentally measured profiles, $\gamma_{\zeta}(\psi)$ can be specified according to experimental observations and modelling outside MHD.

\subsubsection{Pfirsch-Schlüter factor}

The amount of equilibrium flow parallel to the field lines is given by Eq. (16) as $u_{\|} \equiv \mathbf{B} \cdot \mathbf{u} / B=\Phi B / \rho+R \Omega B_{\phi} / B$, which is not a flux function. For a tokamak with a large aspect ratio and circular flux surfaces, using Shafranov-shifted polar coordinates $(r, \theta)$, the flux-surface-average amount of parallel flow is related to the poloidal flow $u_{\theta}$ and toroidal flow $u_{\phi}$ by [52]

$$
\left\langle B u_{\|}\right\rangle=\left\langle B_{\theta} u_{\theta}\right\rangle\left(1+2 q^{2}\right)+\left\langle B_{\phi} u_{\phi}\right\rangle .
$$

where $q=r B_{\phi} / R B_{\theta}$ is the safety factor, introduced in section 3.3.4. An assumption used in the derivation of Eq. (17) is that $\Phi(\psi) / \rho$ is a flux function, which only holds for low flow velocities for which $\rho=\rho_{\mathrm{s}}(\psi)$.

From Eq. (17) we see that the poloidal flow velocity $u_{\theta}$ comes with an unexpected additional factor $1+2 q^{2}$, called the Pfirsch-Schlüter inertial enhancement factor. This can be understood from Eq. (16) by noticing that for plasma to flow in the poloidal direction, it has to move along the magnetic field and then move back toroidally to return to its original poloidal plane. This excursion creates an additional effective inertia for motion in the poloidal direction.

\section{Analytical equilibria with toroidal flow}

The extended Grad-Shafranov equation introduced in the previous section will be solved analytically under simplifying assumptions. To increase the versatility of the thus obtained analytical solutions, various homogeneous solutions that may be added are discussed. Expressions for equilibrium properties like the Mach number, plasma beta, and the safety factor are subsequently derived, allowing control over the characteristics of the solutions. Finally, alternative analytical solutions are considered. 


\subsection{Derivation}

Inserting the pressure from Eq. (10) into the extended Grad-Shafranov Eq. (9), we obtain

$$
\Delta^{*} \psi=-\frac{d \frac{1}{2} F^{2}}{d \psi}-R^{2} \frac{\partial}{\partial \psi}\left[p_{s}(\psi)\left(1+\frac{R^{2} \Omega(\psi)^{2}}{2 \zeta(\psi) T_{s}(\psi)}\right)^{\zeta(\psi)}\right] .
$$

In order to arrive at an analytical solution, we assume $\zeta(\psi)=\zeta$ to be constant and

$$
\frac{d \frac{1}{2} F^{2}}{d \psi}=J, \quad \frac{d p_{s}}{d \psi}=P, \quad \frac{\Omega(\psi)^{2}}{2 T_{s}(\psi)}=\Lambda,
$$

with $J, P$, and $\Lambda$ constants. Inserting these assumptions into Eq. (18) gives

$$
R \frac{\partial}{\partial R}\left(\frac{1}{R} \frac{\partial \psi}{\partial R}\right)+\frac{\partial^{2} \psi}{\partial Z^{2}}=-J-R^{2} P\left(1+\frac{R^{2} \Lambda}{\zeta}\right)^{\zeta} .
$$

This linear partial differential equation is solved by

$$
\psi=c_{0}+c_{1} R^{2}+c_{2} R^{2}\left(Z^{2}-\frac{R^{2}}{4}\right)-\frac{J Z^{2}}{2}-\frac{P \zeta^{2}}{4 \Lambda^{2}} \frac{\left(1+R^{2} \Lambda / \zeta\right)^{\zeta+2}-\left(1+(\zeta+2) R^{2} \Lambda / \zeta\right)}{(\zeta+1)(\zeta+2)},
$$

where the first three terms represent a homogeneous solution and the last two terms form the particular solution. Other solutions that produce the inhomogeneous term $-J$ in Eq. (20) exist, but $-J Z^{2} / 2$ is the most simple one.

The second term in the numerator of the last term is added to ensure that the solution behaves well in the limit $\Lambda \rightarrow 0$. This term is actually a homogeneous solution that may be added or removed freely because it vanishes under the operation of $\Delta^{*}$. For $\Lambda=0$, the last term of Eq. (21) becomes $-P R^{4} / 8$ so that the solution becomes equal to the well-known polynomial Solov'ev solution [3].

The reader is reminded of the fact that when the plasma flow is purely toroidal, one has the freedom to choose an arbitrary non-zero value for $\zeta$. Using $\exp (x) \equiv \lim _{n \rightarrow \infty}(1+x / n)^{n}$, the last term of Eq. (21) becomes in the limit $\zeta \rightarrow \infty$

$$
-\frac{P}{4 \Lambda^{2}}\left(e^{R^{2} \Lambda}-\left(1+R^{2} \Lambda\right)\right) \text {. }
$$

The solutions for $\zeta \rightarrow \infty$ and $\zeta=\gamma /(\gamma-1)$, i.e. with the temperature and the entropy constant on magnetic surfaces respectively, were previously obtained in Ref. [18].

\subsection{Vacuum solutions}

The first three terms of the solution given by Eq. (21) satisfy the vacuum equation $\Delta^{*} \psi_{v}=0$ so that they may be referred to as 'vacuum solutions'. An arbitrary number of vacuum solutions $\psi_{v}$ can be added to Eq. (21), providing additional control over the characteristics of the solution. The poloidal magnetic flux associated with these vacuum solutions may be thought of as originating from external magnetic field coils.

Using separation of variables, $\Delta^{*} \psi_{v}=0$ is solved by $\psi_{v}=f(R) g(Z)$ when $\Delta^{*} f=k f$ and $\Delta^{*} g=-k g$. The first of these equations is Bessel's equation for $f / R$ while the second is the harmonic equation. A general separable vacuum solution can therefore be expressed as

$$
\psi_{v}=R \sum_{n} \cos \left(k_{n} Z\right)\left[a_{n} J_{1}\left(k_{n} R\right)+b_{n} Y_{1}\left(k_{n} R\right)\right],
$$

with $k_{n}, a_{n}, b_{n}$ free constants and $J_{1}$ and $Y_{1}$ the first order Bessel functions of the first and second kind, respectively. We choose to look at up-down symmetric solutions only, which is why in Eq. (23) only cosines were used.

Perhaps more convenient is to use a solution consisting only of polynomials [19]

$$
\psi_{v}=R^{2} \sum_{n=0}^{n_{\max }} a_{n} \sum_{m=0}^{n} \frac{(-1)^{m}(R / 2)^{2(n-m)} Z^{2 m}}{(n-m) !(n-m+1) !(2 m) !} .
$$

The first two terms are the second and third term of Eq. (21). The third term from Eq. (24) is proportional to $R^{2}\left(R^{4}-12 R^{2} Z^{2}+8 Z^{4}\right)$. An alternative non-polynomial third vacuum term is given by [16]

$$
\psi_{v}=c_{3}\left(R^{2} \ln R-Z^{2}\right) .
$$




\subsection{Controlling the characteristics of the solution}

\subsubsection{Shape}

The free constants $c_{1}, c_{2}$ of the solution of Eq. (21) can be used to specify the aspect ratio $\epsilon^{-1} \equiv R_{0} / a$ and the ellipticity $E$ of the solution shown in Fig. 1. When the vacuum solution of Eq. (25) is added to Eq. (21), the third free constant $c_{3}$ can be used to tune the triangularity of the plasma cross-section. To ensure a tokamak configuration, at $Z=0$ the outermost flux surface of the plasma should go through both $R=R_{0}-a$ and $R=R_{0}+a$, i.e. $\psi_{\epsilon}\left(c_{1}, c_{2}\right) \equiv \psi\left(R_{0}+a, 0\right)-\psi\left(R_{0}-a, 0\right)$ should vanish. The value of $c_{1}$ required to ensure $\psi_{\epsilon}=0$ can be obtained from

$$
c_{1}=\frac{-\psi_{\epsilon}\left(c_{1}=0\right)}{\psi_{\epsilon}\left(c_{1}=1\right)-\psi_{\epsilon}\left(c_{1}=0\right)} .
$$

To ensure a certain ellipticity $E$, defined in Fig. 1 , one demands that at $R=R_{0}$ this outermost flux surface additionally goes through $Z=E a$ and $Z=-E a$. Because the solution of Eq. (21) only contains even powers of $Z$, it is top-down symmetric so that when a flux surface goes through one of these points, it automatically goes through the other point. An ellipticity $E$ can therefore be assured by requiring that $\psi_{E}\left(c_{1}, c_{2}\right) \equiv \psi\left(R_{0}, E a\right)-\psi\left(R_{0}+a, 0\right)$ vanishes so that

$$
c_{2}=\frac{-\psi_{E}\left(c_{2}=0\right)}{\psi_{E}\left(c_{2}=1\right)-\psi_{E}\left(c_{2}=0\right)} .
$$

For evaluating both (26a) and (26b), the value of the other coefficient $c_{1}$ or $c_{2}$ is required. It is therefore necessary to evaluate these self-consistently, or evaluate one analytically. The other coefficient can then be calculated either analytically as well, or numerically. Calculating $c_{1}$ from Eqs. (26a) and (21) gives

$$
c_{1}=\frac{1}{2}\left(R_{0}^{2}+a^{2}\right) c_{2}+\frac{P}{16 a R_{0} \Lambda^{2}}(f(a)-f(-a))-\frac{P}{4 \Lambda} \frac{\zeta}{1+\zeta},
$$

where

$$
f(\alpha)=\frac{\zeta^{2}}{(\zeta+1)(\zeta+2)}\left(1+\left(R_{0}+\alpha\right)^{2} \Lambda / \zeta\right)^{\zeta+2} .
$$

In the limit $\zeta \rightarrow \infty$, this becomes $f(\alpha)=\exp \left(\left(R_{0}+\alpha\right)^{2} \Lambda\right)$. Substituting Eq. (27) into Eq. (26b) gives

$$
c_{2}=\frac{1}{a^{2} R_{0}^{2}\left(1-\left(a / 2 R_{0}\right)^{2}+E^{2}\right)}\left[J \frac{(a E)^{2}}{2}+\frac{P}{4 \Lambda^{2}}\left(\frac{f(a)-f(-a)}{2}\left(1+\frac{a}{2 R_{0}}\right)+f(0)-f(a)\right)\right] .
$$

With this result inserted into Eq. (27) one has the closed form expressions for $c_{1}$ and $c_{2}$ ensuring a closed flux surface through $\left(R_{0} \pm a, 0\right)$ and $(0, \pm E a)$. To make the expressions dimensionless one may choose either $R_{0}=1$ and $a=\epsilon$, or $R_{0}=\epsilon^{-1}$ and $a=1$. To control the triangularity one can add another vacuum solution proportional to $c_{3}$, e.g. Eq. (25), and follow the same procedure as outlined above to find $c_{3}$. Note that this does introduce additional terms in the above expressions for $c_{1}$ and $c_{2}$.

\subsubsection{The Mach number}

The assumptions of Eq. (19) impose restrictions on the kind of equilibria that the analytical solution can describe. The function $F$ is usually approximately constant for tokamaks, with poloidal currents creating a small 'diamagnetic dip' that can be described quite well with $F^{2}$ an offset-linear function of $\psi$. For a large aspect ratio tokamak plasma, the poloidal flux function $\psi$ is an approximately quadratic function of the distance to the magnetic axis, implying that a function linear in $\psi$ can describe approximately parabolic profiles. The second assumption of Eq. (19) therefore restricts the pressure profile to be approximately parabolic, which may be too smooth to describe certain tokamak regimes. The third assumption states that the angular frequency goes down as the square root of the static temperature $T_{s}$. This assumption has an interesting implication for the Mach number $M \equiv R \Omega / \sqrt{\gamma T}=R \sqrt{2 \Lambda T_{s} / \gamma T}$ which, using Eqs. (10) and (11), can be written as

$$
M=\frac{R \sqrt{2 \Lambda / \gamma}}{\sqrt{1+R^{2} \Lambda / \zeta}} .
$$

Note that the Mach number depends only on $R$. The denominator of Eq. (30) places a limit on the maximum Mach number that can be described. In the limit $\Lambda \rightarrow \infty$ we obtain

$$
M_{\max }=\sqrt{2 \zeta / \gamma}
$$

For isothermal flux surfaces $M=R \sqrt{2 \Lambda / \gamma}$, so that there is no limit on $M$.

\subsubsection{The plasma beta}

A parameter often used to characterize a tokamak plasma is the ratio $\beta \equiv 2 p / B^{2}$ between the pressure and the magnetic pressure. With the efficiency of a reactor increasing with increasing $p$ and its cost increasing with increasing $B^{2}, \beta$ is an important parameter to roughly judge the performance of a tokamak as a power plant. A high value of $\beta$ is desirable, 
instabilities limit the currently achievable value to only a few percent. Typically the toroidal vacuum magnetic field $B_{0}$ at the geometrical axis and an average pressure $\langle p\rangle$ are used. Sometimes however an average $\beta$ is used, defined as

$$
\langle\beta\rangle \equiv 2 \frac{\int p d V}{\int B^{2} d V},
$$

where the integration is performed over the plasma volume. From Eq. (4a), using partial integration, and finally the extended Grad-Shafranov Eq. (9),

$$
\int B^{2} d V=\int \frac{F^{2}+|\nabla \psi|^{2}}{R^{2}} d V=\int \frac{F^{2}-\left(\psi-\psi_{e}\right) \Delta^{*} \psi}{R^{2}} d V=\int\left(\left(\psi-\psi_{e}\right)\left(\frac{\partial p}{\partial \psi}+\frac{1}{R^{2}} \frac{d \frac{1}{2} F^{2}}{d \psi}\right)+\frac{F^{2}}{R^{2}}\right) d V,
$$

where $\psi_{e}$ denotes the value of $\psi$ at the edge so that the divergence term $\int \nabla \cdot\left(R^{-2}\left(\psi-\psi_{e}\right) \nabla \psi\right) d V$ vanished. When the pressure at the plasma edge vanishes we can use the assumptions (19) to write $\left(\psi-\psi_{e}\right) \partial p / \partial \psi=p$ and $\left(\psi-\psi_{e}\right) d F^{2} / d \psi=F^{2}-F_{e}^{2}$, where $F_{e}$ denotes the value of $F$ at the plasma edge. By Ampere's law Eq. (1e), the magnitude of the toroidal vacuum field is given by $B_{0} R_{0} / R$ so that we have $F_{e} \equiv B_{\phi e} R_{e}=B_{0} R_{0} \equiv F_{0}$. The average $\beta$ can then be written as

$$
\langle\beta\rangle=\frac{2 \int p d V}{\int\left(p+\frac{1}{2} R^{-2}\left(3 F^{2}-F_{0}^{2}\right)\right) d V} .
$$

The integrals in Eq. (34) have to be calculated numerically in general. When $R_{0}^{2} \Lambda \ll 1$, the primary 'knob' to tune $\langle\beta\rangle$ is the ratio between the pressure gradient $P$ and $3 F^{2}-F_{0}^{2}$. For higher flow velocities, $\Lambda$ plays an important role as well.

\subsubsection{The safety factor}

Another important parameter to characterize a tokamak equilibrium is the safety factor which, with $B_{p}$ the poloidal magnetic field, is defined by

$$
q \equiv \frac{1}{2 \pi} \oint \frac{B_{\phi}}{R B_{p}} d l=\frac{F(\psi)}{2 \pi} \oint \frac{d l}{R|\nabla \psi|},
$$

where in the second step we used $R B_{p}=|\nabla \psi|$ and $R B_{\phi}=F(\psi)$ from Eq. (4a). The integration path of Eq. (35) is along the poloidal magnetic field over one full poloidal turn. With $B_{\phi} / B_{p}$ the local pitch of the magnetic field line, $q$ gives the number of toroidal turns per poloidal turn of the magnetic field. Since the magnetic field lines do not cross, $q=q(\psi)$ is a flux function.

From Eq. (5) we have $2 \pi d \psi=B_{p} d A_{t}$ for the infinitesimal poloidal flux through an annulus of area $d A_{t}=2 \pi R d R$. The infinitesimal toroidal flux through a poloidal cross-section of area $d A_{p}=\oint d R d l$ is similarly given by $2 \pi d \chi=B_{\phi} d A_{p}$ so that

$$
q(\psi)=\frac{d \chi}{d \psi}=\frac{B_{\phi}}{2 \pi} \frac{d A_{p}}{d \psi} .
$$

Taylor expanding $\psi(R, Z)$ to second order around the magnetic axis, where $\nabla \psi$ vanishes, yields flux surfaces with elliptical poloidal cross-sections. The cross-sectional areas satisfy $d A_{p} / d \psi=2 \pi / \sqrt{D}$ with $D=\operatorname{det}(\nabla \nabla \psi)=\psi_{R R} \psi_{Z Z}-\psi_{R Z}^{2}$ the discriminant, the determinant of the Hessian. The safety factor at the magnetic axis is therefore given by

$$
q\left(\psi_{m}\right)=\frac{B_{\phi}}{\sqrt{\psi_{R R} \psi_{Z Z}-\psi_{R Z}^{2}}} .
$$

For up-down symmetric equilibria like the analytical equilibria of Eq. (21), $\psi_{R Z}$ vanishes at the magnetic axis. From Eq. (21), we have at the magnetic axis $\psi_{\mathrm{Zz}}=2 c_{2} R_{m}^{2}-J$ and

$$
\psi_{R R}=2 c_{1}-3 c_{2} R_{m}^{2}+\frac{P}{2 \Lambda} \frac{\zeta}{\zeta+1}\left[1-\left(1+\frac{R_{m}^{2} \Lambda}{\zeta}\right)^{\zeta}\left(1+\frac{R_{m}^{2} \Lambda}{\zeta}(3+2 \zeta)\right)\right] .
$$

Note that the equation $d \psi(R, 0) / d R=0$ for the position of the magnetic axis, with $\psi(R, Z)$ from Eq. (21), cannot be solved analytically for general $\zeta$. In the limit $\zeta \rightarrow 1$, however, one finds

$$
R_{m}^{2}=\frac{-P-2 c_{2}+\sqrt{\left(P+2 c_{2}\right)^{2}+8 c_{1} P \Lambda}}{P \Lambda} .
$$

\subsection{Other analytical solutions}

Eq. (18) can be solved analytically for other assumptions than those of Eq. (19). When we assume that $d \frac{1}{2} F^{2} / d \psi$ is given by $K \psi$ instead of by $J$, the extended Grad-Shafranov Eq. (18) becomes

$$
\left(\Delta^{*}+K\right) \psi=-R^{2} P\left(1+R^{2} \Lambda / \zeta\right)^{\zeta},
$$


which has the solution [19]

$$
\psi=-\frac{P R}{K^{3 / 2}} \sum_{n=0}^{\infty} \frac{\zeta}{n}\left(\frac{\Lambda}{\zeta K}\right)^{n} S_{2 n+2,1}(\sqrt{K} R)+R \cos \left(k_{n} Z\right)\left[a_{n} J_{1}\left(R \sqrt{K-k_{n}^{2}}\right)+b_{n} Y_{1}\left(R \sqrt{K-k_{n}^{2}}\right)\right],
$$

where $a_{n}, b_{n}$ and $k_{n}$ are free constants. The first part of Eq. (41) represents a particular solution in terms of Lommel functions $S_{\mu, v}$ and the second part represents a general homogeneous solution in terms of Bessel functions. This solution has the same maximum Mach number as the solution of Eq. (21) given in Eq. (31), but has a more peaked toroidal current density. An obvious drawback for practical applications is the evaluation of an infinite sequence of special functions.

Perhaps a more useful analytical solution is obtained assuming additionally that $p_{s}^{\prime}=Q \psi$ instead of $P$, so that Eq. (18) becomes

$$
\left(\Delta^{*}+K+Q^{2} R^{2}\left(1+R^{2} \Lambda / \zeta\right)^{\zeta}\right) \psi=0 .
$$

which is solved by [19]

$$
\psi=\sqrt{1+R^{2} \Lambda / \zeta} \cos (\sqrt{K} Z)\left(a J_{1 /(\zeta+2)}(\tau)+b Y_{1 /(\zeta+2)}(\tau)\right)
$$

where $\tau=(\sqrt{2 Q} / \Lambda)(\zeta /(\zeta+2))\left(1+R^{2} \Lambda / \zeta\right)^{(\zeta+2) / 2}$. A property of this solution is that the toroidal current $J_{\phi}=\Delta^{*} \psi / R$ is proportional to $\psi$. The toroidal current can therefore be made to vanish at the plasma edge, by making $\psi$ vanish there.

\section{The Brunt-Väisälä frequency}

The gravitational stability of an adiabatically displaced air parcel in the atmosphere depends on the vertical stratification of pressure and density. Something similar holds for plasma under the influence of the centrifugal force. By analogy, in Section 4.2.2 expressions are derived for the oscillation frequency of plasma confined to the circular magnetic surfaces of a large aspect ratio tokamak.

\subsection{In the atmosphere}

Imagine a horizontally stratified atmosphere in which one displaces a fluid parcel a distance $z$ in the vertical $\hat{\mathbf{z}}$-direction, from position 1 to position 2 . The parcel, arrived at the final position 2 , will not have the same thermodynamic variables as its new environment, so that we will denote these with a subscript $f$. When the parcel is displaced sufficiently fast there will be no heat exchange with the environment and the process will be adiabatic, i.e. $S_{f}=S_{1}$. The pressure however equilibrates much faster, so that we can assume $p_{f}=p_{2}$. Therefore the final density $\rho_{f}=\left(p_{f} / S_{f}\right)^{1 / \gamma}=\left(p_{2} / S_{1}\right)^{1 / \gamma}$ will differ from the ambient density $\rho_{2}$. With $g<0$, this density difference results in a buoyancy force $g\left(\rho_{f}-\rho_{2}\right) \hat{\mathbf{z}}$. We can set up the equation of motion for the vertical position of the fluid parcel

$$
\frac{d^{2} z}{d t^{2}}=g \frac{\rho_{f}-\rho_{2}}{\rho_{f}}=g \frac{S_{1}^{-1 / \gamma}-S_{2}^{-1 / \gamma}}{S_{1}^{-1 / \gamma}} \approx \frac{g}{\gamma} \frac{1}{S} \frac{d S}{d z} z
$$

The resulting frequency of the exponential solutions $z \propto$ expi $\omega_{\mathrm{BV}} t$ is called the buoyancy frequency, or Brunt-Väisälä (BV) frequency

$$
\omega_{\mathrm{BV}}^{2} \equiv-\frac{g}{\gamma} \frac{S^{\prime}}{S}=g\left(\frac{\rho^{\prime}}{\rho}-\frac{p^{\prime}}{\gamma p}\right)
$$

where a prime denotes differentiation with respect to $z$. When this squared frequency is positive, the buoyancy force is restoring and the atmosphere is stable. When however

$$
g\left(\frac{\rho^{\prime}}{\rho}-\frac{p^{\prime}}{\gamma p}\right)<0
$$

instability will result. This criterion for convective instability is known as the Schwarzschild criterion.

When the fluid parcel is displaced sufficiently slow for heat exchange with the ambient air to take place, the temperature will equilibrate as well. In this case there will no longer be a density difference with the environment, no restoring force, and no oscillations or instability.

When the fluid parcel is displaced without being able to compress, its final density will be $\rho_{f}=\rho_{1}$ so that

$$
\omega_{\mathrm{BV}, \text { incompr. }}^{2}=g \frac{\rho^{\prime}}{\rho} \text {. }
$$

\subsection{In a tokamak}

For a tokamak plasma, the issue of gravitational stability discussed above is not very relevant. Centrifugal forces however can be significant in a tokamak. Like the gravitational force, the centrifugal force is proportional to the density. In Eq. (3), the 
effective gravitational acceleration is given by $g=R \Omega^{2}$ in the $R$-direction. Because of the presence of the magnetic field, the plasma is highly anisotropic. We therefore separately discuss the centrifugal convective effect on plasma perturbations normal to the magnetic surfaces and within the magnetic surfaces. In both instances, the perturbation needs to have at least a component in the direction of the centrifugal force.

\subsubsection{Normal to the magnetic surfaces}

Perturbations perpendicular to the magnetic surfaces are to a good approximation incompressible, in order to minimize the large energy associated with the compression of magnetic field lines. We consider a plasma perturbation in the horizontal $Z=0$ plane. Using $g=R \Omega^{2}$ in Eq. (47) yields

$$
\omega_{\mathrm{BV}, \perp}^{2}=\frac{R \Omega^{2} \rho^{\prime}}{\rho} .
$$

This frequency squared combines with that associated with the magneto-rotational instability $\omega_{\mathrm{MRI}}^{2}=R\left(\Omega^{2}\right)^{\prime}[53-55]$ to give [56]

$$
\rho \omega^{2}=\rho\left(\omega_{\mathrm{BV}, \perp}^{2}+\omega_{\mathrm{MRI}}^{2}\right)=R\left(\rho \Omega^{2}\right)^{\prime} .
$$

\subsubsection{Within the magnetic surfaces}

Within the magnetic surfaces, plasma perturbations with a component in the $R$-direction will also experience the convective effect. Contrary to the perturbations in the $\nabla \psi$-direction, these perturbations can be compressible and will oscillate in the presence of entropy gradients as described by Eq. (45). The relevant entropy gradients are those within the magnetic surfaces, projected on the direction of the centrifugal force, i.e.

$$
\left.\frac{\partial S}{\partial R}\right|_{\psi}=\left.\frac{\partial}{\partial R}\right|_{\psi} p_{s} \rho_{s}^{-\gamma}\left(1+\frac{R^{2} \Lambda}{\zeta}\right)^{\zeta-\gamma(\zeta-1)}=S\left(1-\frac{\gamma}{\gamma_{\zeta}}\right) \frac{2 R \Lambda}{1+R^{2} \Lambda / \zeta},
$$

where we used $S=p \rho^{-\gamma}$ with $p$ and $\rho$ from Eqs. (10) and (11) and we used the definition $\gamma_{\zeta}(\psi) \equiv \zeta /(\zeta-1)$. Plasma moving along a magnetic field line has to move in the toroidal as well as the poloidal direction in order to move in the $R$-direction. This increases the effective inertia by the Pfirsch-Schlüter factor $1+2 q^{2}$ as described in Section 2.3.2. The BV-frequency is then decreased by one over this factor. For circular flux-surfaces another factor of $1 / 2$ accounts for the average directional $\left\langle(\hat{\boldsymbol{\theta}} \cdot \nabla R)^{2}\right\rangle=\left\langle\cos ^{2} \theta\right\rangle=1 / 2$ resulting from the fact that the plasma is restrained to move in the poloidal $\hat{\boldsymbol{\theta}}$-direction while both the centrifugal force and the entropy gradients are in the $\nabla R$-direction. From Eq. (45), with $g=R \Omega^{2}$, we can then define the average BV-frequency of a tokamak with circular flux-surfaces as

$$
\omega_{\mathrm{BV}, \text { tokamak }}^{2} \equiv-\left.\frac{R \Omega^{2}}{2\left(1+2 q^{2}\right)} \frac{1}{\gamma S} \frac{\partial S}{\partial R}\right|_{\psi}=\frac{\gamma / \gamma_{\zeta}-1}{1+R^{2} \Lambda / \zeta} \frac{R^{2} \Lambda \Omega^{2} / \gamma}{1+2 q^{2}}=\left(\frac{\gamma}{\gamma_{\zeta}}-1\right) \frac{M^{2} \Omega^{2}}{2\left(1+2 q^{2}\right)},
$$

with $M$ given by Eq. (30). As discussed in Section 2.3.1 the adiabatic constants $\gamma_{\zeta}$ and $\gamma$ may be different, leading to stable oscillations when $\gamma_{\zeta}<\gamma$ or instability when $\gamma_{\zeta}>\gamma$.

\section{Waves and instabilities}

Up to now we have been discussing plasma configurations in which the Lorentz force, the pressure force, and the centrifugal force are balanced. We do not yet know if these configurations are stable against slight deviations from equilibrium. A small perturbation may create a restoring force, resulting in a wave within the plasma. The resulting force inequilibrium caused by the perturbation may however also amplify the original perturbation, leading to instability.

First, the Eulerian approach to stability is introduced that is implemented in the numerical code of the next section. Next, a Lagrangian approach is described and used to obtain an expression for the potential energy of a plasma perturbation. Finally, an analytical expression for the low-frequency continuous Alfvén spectrum is obtained. Both these expressions contain the freedom to choose which thermodynamic quantity is constant on the equilibrium magnetic surfaces and in both the BVfrequency of the previous section plays a role. As a primer, the continuous spectrum in an infinite homogeneous plasma and a cylinder are briefly revisited.

\subsection{Primitive variables}

In order to investigate the waves and instabilities in an equilibrium, one can split all quantities in an equilibrium value plus a perturbation. Inserting these quantities into the time-dependent ideal MHD equations, linear stability is investigated by retaining only terms that depend on the perturbations to first order. Because the resulting linear equations do not depend on time explicitly, one can assume normal mode solutions in which the perturbations depend on time harmonically, i.e. they are proportional to $\exp (-i \omega t)$. The entire system of equations can then be cast into an eigenvalue problem for the 
eigenvalues $\omega$. This procedure is typically followed in numerical codes, where the radial and poloidal directions are handled with finite elements and/or spectral methods. In the case of axisymmetry, the perturbations $\delta f$ of the primitive physical variables $f=p, \rho, \mathbf{B}, \mathbf{u} \ldots$ are typically parametrized as

$$
\delta f(\psi, \vartheta, \phi, t)=\sum_{m} \delta f_{m}(\psi) e^{i(m \vartheta-\omega t-n \phi)},
$$

where $\vartheta$ is some poloidal coordinate and the integers $m$ and $n$ are the poloidal and toroidal mode number, respectively. Because the equations do not depend on the toroidal coordinate $\phi$ explicitly, one can investigate single normal modes in the toroidal direction. The symmetry in the poloidal direction is not exact, so that in general several poloidal mode numbers have to be included in Eq. (52).

\subsection{Plasma perturbation}

Another approach is to start from an infinitesimal displacement $\xi$ of the plasma. This perturbation automatically also perturbs other quantities like the magnetic field, the pressure, and the density

$$
\begin{aligned}
& \delta \mathbf{B}=\nabla \times(\boldsymbol{\xi} \times \mathbf{B}), \\
& \delta \rho=-\nabla \cdot(\rho \xi), \\
& \delta p=-\xi \cdot \nabla p-\gamma p \nabla \cdot \xi,
\end{aligned}
$$

These Eulerian perturbations follow from Eqs. (1d) and (1g), Eq. (1b), and Eqs. (1c) and (12), respectively. Inserting the perturbed quantities into the static (i.e. without flow) time-dependent ideal MHD equations gives an equation of motion for $\xi$

$$
\rho \ddot{\xi}=\mathbf{F}_{s}(\xi)
$$

where the static force operator is given by [57]

$$
\mathbf{F}_{s}(\boldsymbol{\xi})=((\nabla \times \delta \mathbf{B}) \times \mathbf{B}+(\nabla \times \mathbf{B}) \times \delta \mathbf{B}-\nabla \delta p .
$$

The first two terms can be recognized as the linearised perturbed Lorentz force and the last term as the perturbed pressure force. Assuming a harmonic time dependence, Eq. (54) becomes an eigenmode equation $\ddot{\xi}=-\omega^{2} \xi$ for the eigenvalues $\omega^{2}$. It can be shown that $\mathbf{F}_{s}$ is a Hermitian operator so that the eigenvalues $\omega^{2}$ are real. This implies that the frequencies $\omega$ are either purely real, corresponding to stable waves, or purely imaginary, corresponding to instabilities.

\subsection{Frieman-Rotenberg approach}

In a formalism developed by Frieman and Rotenberg [58], a Lagrangian approach is taken in which $\xi$ describes the position of the perturbed plasma element relative to the background flow. Assuming $\xi$ to be of the form (52), the equation of motion changes from the static Eq. (54) to give [58]

$$
-\rho \omega \xi-2 i \rho \omega \mathbf{u} \cdot \nabla \boldsymbol{\xi}=\mathbf{F}(\boldsymbol{\xi})
$$

where the total force operator $\mathbf{F}=\mathbf{F}_{s}+\mathbf{F}_{u}$, with a rotational force $\mathbf{F}_{u}=\nabla \cdot(\rho \xi \mathbf{u} \cdot \nabla \mathbf{u}-\rho \mathbf{u u} \cdot \nabla \xi)$. The second term in Eq. (56) can be recognized as resulting from the Coriolis effect. work

For a toroidally rotating equilibrium with velocity $\mathbf{u}=R \Omega \hat{\boldsymbol{\phi}}$ and angular frequency vector $\boldsymbol{\Omega}=-\Omega \widehat{\mathbf{Z}}$, we obtain after some

$$
\mathbf{F}_{u}=-\mathbf{R} \nabla \cdot\left(\rho \Omega^{2} \boldsymbol{\xi}\right)+n^{2} \rho \Omega^{2} \boldsymbol{\xi}+2 i n \rho \Omega(\boldsymbol{\Omega} \times \boldsymbol{\xi}) .
$$

Eq. (56) can then be rewritten in terms of the Doppler shifted frequencies $\omega_{D}=\omega+n \Omega$ as [59]

$$
-\rho \omega_{D}^{2} \boldsymbol{\xi}-2 i \rho \omega_{D} \boldsymbol{\Omega} \times \boldsymbol{\xi}=\mathbf{F}_{s}(\boldsymbol{\xi})-\mathbf{R} \nabla \cdot\left(\rho \Omega^{2} \boldsymbol{\xi}\right)
$$

\subsection{Stability}

The equation of motion, Eqs. (56) or (58), is no longer a standard eigenvalue equation because the eigenvalue $\omega$ appears both linearly and quadratically. This allows the frequencies to become complex-valued. Taking the inner product of Eqs. (56) or (58) with $\xi^{*}$ and integrating over the entire plasma volume gives, solving for $\omega[58,60]$

$$
\omega=\bar{V} \pm \sqrt{\bar{V}^{2}+\bar{W}}
$$

where $V=-\frac{1}{2} \int \rho\left(n \Omega|\xi|^{2}+i \xi^{*} \cdot(\mathbf{\Omega} \times \xi)\right) d^{3} \mathbf{r}$ is the Doppler-Coriolis shift, $W=-\frac{1}{2} \int \xi^{*} \cdot \mathbf{F} d^{3} \mathbf{r}$ the potential energy, and a bar denotes normalization with $I \equiv \frac{1}{2} \int \rho|\xi|^{2} d^{3} \mathbf{r}$. The frequencies of Eq. (59) are real when $\bar{W}>-\bar{V}^{2}$, providing a stability criterion for toroidally rotating tokamak plasmas. 
When the rotation is rigid, or can be considered rigid because the flow shear is insignificant over the area where the mode amplitude is nonzero, $\omega_{D}$ is (locally) constant. Taking the inner product of Eq. (58) with $\xi^{*}$ and integrating over the entire plasma volume gives

$$
\omega_{D}=\overline{\mathcal{V}} \pm \sqrt{\overline{\mathcal{V}}^{2}+\overline{\mathcal{W}}}
$$

where $\mathcal{V}=-\frac{1}{2} \int i \rho \xi^{*} \cdot \mathbf{\Omega} \times \xi d^{3} \mathbf{r}$ and $\mathcal{W}=-\frac{1}{2} \int \xi^{*} \cdot\left(\mathbf{F}_{s}(\xi)-\mathbf{R} \nabla \cdot\left(\rho \Omega^{2} \xi\right)\right) d^{3} \mathbf{r}$. The frequencies are real when $\overline{\mathcal{W}}>-\overline{\mathcal{V}}^{2}$, providing a necessary and sufficient stability criterion for rigidly rotating tokamak plasmas.

\subsection{Potential energy}

The potential energy $W=-\frac{1}{2} \int \xi^{*} \cdot \mathbf{F} d^{3} \mathbf{r}$ can be rewritten as [61]

$$
W=\frac{1}{2} \int\left(|\delta \mathbf{B}|^{2}+\mathbf{J} \cdot \xi^{*} \times \delta \mathbf{B}+U_{n}+U\right) d^{3} \mathbf{r},
$$

where the manifestly $n$-dependent part of the rotational potential energy density is given by

$$
U_{n}=-\xi^{*} \cdot\left(n^{2} \rho \Omega^{2} \xi+2 i n \rho \Omega \boldsymbol{\Omega} \times \xi\right)=-n^{2} \rho \Omega^{2}|\xi|^{2}-4 i n \rho \Omega \boldsymbol{\Omega} \cdot \operatorname{Re}(\xi) \times \operatorname{Im}(\xi)
$$

and the remaining non-magnetic part of the potential energy density

$$
U \equiv-\left(\nabla \cdot \xi^{*}\right) \delta p+\xi^{*} \cdot \mathbf{R} \nabla \cdot\left(\rho \Omega^{2} \xi\right) .
$$

where the divergence $\nabla \cdot\left(\xi^{*} \delta p\right)$ was discarded since, for internal modes, this will give a vanishing surface term when integrated over the entire plasma volume. To investigate the influence of toroidal rotation on stability, we take a closer look at $U$,

$$
U=\left(\nabla \cdot \xi^{*}\right)(\xi \cdot \nabla p+\gamma p \nabla \cdot \xi)+\xi^{*} \cdot \mathbf{R}\left(\rho \Omega^{2} \nabla \cdot \xi+\xi \cdot \nabla\left(\rho \Omega^{2}\right)\right) .
$$

Writing in components $\xi \cdot \nabla=\xi_{\psi} \partial / \partial \psi+\xi_{R} \partial / \partial R+\xi_{\phi} \partial / \partial \phi$, we have

$$
\xi \cdot \nabla p=\xi_{\psi} \frac{\partial p}{\partial \psi}+\xi_{R} \rho R \Omega^{2},
$$

where the force balance Eq. (8) was used to replace $\partial p / \partial R$ by $\rho R \Omega^{2}$. To rewrite the last term of Eq. (64), we use

$$
\frac{\partial \rho \Omega^{2}}{\partial R}=\frac{\zeta-1}{\zeta} \frac{\rho \Omega^{2}}{p} \frac{\partial p}{\partial R}=\frac{R\left(\rho \Omega^{2}\right)^{2}}{\gamma_{\zeta} p},
$$

where we used $\gamma_{\zeta} \equiv \zeta /(\zeta-1)$ and Eq. (14) for $\rho$. Using Eqs. (65) and (66) in Eq. (64) gives

$$
U=\left(\nabla \cdot \xi^{*}\right)\left(\xi_{\psi} \frac{\partial p}{\partial \psi}+\xi_{R} \rho R \Omega^{2}+\gamma p \nabla \cdot \xi\right)+R \xi_{R}^{*}\left(\rho \Omega^{2} \nabla \cdot \xi+\xi_{\psi} \frac{\partial \rho \Omega^{2}}{\partial \psi}+\xi_{R} \frac{R\left(\rho \Omega^{2}\right)^{2}}{\gamma_{\zeta} p}\right) .
$$

Or, rearranging terms

$$
U=\left(\nabla \cdot \xi^{*}\right) \xi_{\psi} \frac{\partial p}{\partial \psi}+R \xi_{R}^{*} \xi_{\psi} \frac{\partial \rho \Omega^{2}}{\partial \psi}+\gamma p\left|\nabla \cdot \xi+\frac{\rho \Omega^{2}}{\gamma p} R \xi_{R}\right|^{2}+\left(\frac{\gamma}{\gamma_{\zeta}}-1\right) \frac{\left|\rho \Omega^{2} R \xi_{R}\right|^{2}}{\gamma p} .
$$

The component of $\xi$ parallel to the magnetic field is only present in the last two terms. For static equilibria these terms reduce to $\gamma p|\nabla \cdot \xi|^{2}$. In order to minimize $U$, the parallel displacement of modes whose phase velocity is smaller than the thermal velocity will ensure incompressibility of the perturbations. For supersonic modes, the parallel component will be negligible. The additional inertia associated with the parallel motion of subsonic modes will lower the frequency squared of subsonic modes by the Pfirsch-Schlüter factor. In the presence of significant toroidal flow however, the last two terms in Eq. (68) contain the parallel displacement, complicating things somewhat more.

The second term on the right-hand side of Eq. (68) should be compared with Eq. (49) resulting from a combination of the magneto-rotational instability and the convective effect of incompressible radial perturbations $\xi_{\psi}$. This combination was previously found in both astrophysical $[55,62]$ and tokamak plasmas [63,64].

Note that the last term of Eq. (68) can be written as $\left(\gamma / \gamma_{\zeta}-1\right) \rho M^{2} \Omega^{2}\left|\xi_{R}\right|^{2}$. Comparing this with Eq. (51) we have clearly identified this term as originating from the convective effect.

\subsection{Continuous spectrum}

For certain frequencies, common to a magnetic surface, the ideal MHD equations become singular. The associated eigenfunctions show singular behavior at the corresponding magnetic surface. These singular eigenmodes can be thought of as being confined to their respective magnetic surface. Their amplitude will be non-zero over some surrounding region though. Because of the discontinuous behavior of the eigenfunctions, the boundary conditions can always be satisfied so that each of 
these frequencies will be an eigenfrequency. The continuum of magnetic surfaces in this way traces out a continuum of frequencies, called the continuous spectrum [61].

The continuum modes in the presence of a one-dimensional inhomogeneity, as in a cylinder, closely resemble those waves in a homogeneous medium that have their wavevector perpendicular to the equilibrium magnetic field. As a primer to the continuous spectrum of a torus, we will shortly investigate these cases subsequently.

\subsubsection{Infinitely extended homogeneous plasma at rest}

MHD waves can most easily be described in an infinitely extended fluid at rest, with constant equilibrium variables $\mathbf{u}=0$, B, $p, \rho$ [61]. The ideal MHD Eqs. (2a)-(2e) are in this case trivially satisfied, since all gradients vanish. Assuming plane wave solutions for a perturbation $\xi$ with a spatial and time dependence proportional to $\exp (i \mathbf{k} \cdot \mathbf{r}-i \omega t)$, we can replace $\nabla$ with $i \mathbf{k}$ and $\partial / \partial t$ with $-i \omega$. The equation of motion Eq. (54) for a homogeneous background medium becomes

$$
-\rho \omega^{2} \boldsymbol{\xi}=(i \mathbf{k} \times \delta \mathbf{B}) \times \mathbf{B}-i \mathbf{k} \delta p .
$$

Inserting $\delta \mathbf{B}=i \mathbf{k} \times(\xi \times \mathbf{B})$ and $\delta p=-i \gamma p \mathbf{k} \cdot \boldsymbol{\xi}$ from Eqs. (53a) and (53c), and defining $v_{s} \equiv \sqrt{\gamma p / \rho}$ and $\mathbf{v}_{A} \equiv \mathbf{B} / \sqrt{\rho}$, we obtain

$$
-\omega^{2} \boldsymbol{\xi}+\left(\mathbf{k} \times\left(\mathbf{k} \times\left(\xi \times \mathbf{v}_{A}\right)\right)\right) \times \mathbf{v}_{A}+v_{s}^{2} \mathbf{k}(\mathbf{k} \cdot \xi)=0 .
$$

Defining $k_{\|} \equiv \mathbf{k} \cdot \hat{\mathbf{v}}_{A}$, the component of Eq. (70) parallel to $\hat{\mathbf{v}}_{A}$ gives $\omega^{2} \boldsymbol{\xi} \cdot \hat{\mathbf{v}}_{A}=k_{\|} v_{s}^{2} \mathbf{k} \cdot \boldsymbol{\xi}$. Using this relation to eliminate $\boldsymbol{\xi} \cdot \hat{\mathbf{v}}_{A}$ and working out the triple cross-product, Eq. (70) can be written as

$$
\left(\omega^{2}-k_{\|}^{2} v_{A}^{2}\right) \xi+(\mathbf{k} \cdot \xi)\left[\left(v_{A}^{2} v_{s}^{2}\left(\frac{k_{\|}}{\omega}\right)^{2}-\left(v_{s}^{2}+v_{A}^{2}\right)\right) \mathbf{k}+k_{\|} v_{A}^{2} \hat{\mathbf{v}}_{A}\right]=0 .
$$

The component of Eq. (71) perpendicular to both $\hat{\mathbf{v}}_{A}$ and $\mathbf{k}$ gives the Alfvén wave frequencies $\omega_{A}^{2}=k_{\|}^{2} v_{A}^{2}$. The component of Eq. (71) parallel to $\mathbf{k}$ gives the slow $(-)$ and fast $(+)$ magnetosonic wave frequencies

$$
\omega_{s, f}^{2}=k^{2}\left(\frac{v_{s}^{2}+v_{A}^{2}}{2} \pm \sqrt{\left(\frac{v_{s}^{2}+v_{A}^{2}}{2}\right)^{2}-v_{s}^{2} v_{A}^{2}\left(\frac{k_{\|}}{k}\right)^{2}}\right) .
$$

These wave frequencies satisfy $\omega_{f}^{2} \geqslant \omega_{A}^{2} \geqslant \omega_{s}^{2} \geqslant 0$, reflecting the strengths of the restoring forces of these waves. For fast and slow magnetosonic waves, both the plasma pressure and the magnetic pressure are disturbed; in phase and out of phase, respectively. For Alfvén waves, these pressures are not perturbed and the magnetic field line tension acts as a restoring force.

When $k_{\|} \ll k$, Eq. (72) can be expanded to give

$$
\omega_{s}^{2} \approx k_{\|}^{2} \frac{v_{s}^{2} v_{A}^{2}}{v_{s}^{2}+v_{A}^{2}}=\omega_{A}^{2} \frac{v_{s}^{2}}{v_{s}^{2}+v_{A}^{2}}=\omega_{A}^{2} \frac{\gamma p}{\gamma p+B^{2}} \quad \text { and } \quad \omega_{f}^{2} \approx k^{2}\left(v_{s}^{2}+v_{A}^{2}\right) .
$$

We find that the frequencies of both the Alfvén and slow magnetonic waves vanish when $k_{\|}=0$. Because the frequency of the fast wave always remains finite and relatively high, it is of little interest for stability and we will not consider this wave in what follows.

\subsubsection{A cylinder}

When inhomogeneities are introduced, and $\beta \| 1$, the continuum waves that arise satisfy $k_{\|} \ll k$. The expressions of Eq. (73) can therefore be immediately used to describe the continuum of a cylindrical plasma. In a cylinder, all quantities only depend on the radial coordinate $r$ so that single poloidal harmonics $f \propto \operatorname{expi}(m \theta-\omega t-k z)$ can be investigated separately. The Alfvén continuum frequencies are then given by

$$
\omega_{A, m}(r)= \pm k_{\|} v_{A}= \pm \frac{\mathbf{B} \cdot \mathbf{k}}{\sqrt{\rho}}= \pm \frac{\mathbf{B} \cdot \nabla}{i \sqrt{\rho}} e^{i(m \theta-\omega t-k z)}= \pm \frac{k B_{z}-m B_{\theta} / r}{\sqrt{\rho}}= \pm \bar{\omega}_{A}\left(n-\frac{m}{q(r)}\right) .
$$

In the last expression we introduced $q(r)=r B_{z} / R_{0} B_{\theta}, \bar{\omega}_{A}(r)=B_{z} / \sqrt{\rho} R_{0}$ and $n=k R_{0}$ to establish the correspondence between a periodic cylinder with length $2 \pi R_{0}$ and a large aspect ratio torus with cylindrical cross-section. In this case $n$ becomes the integer toroidal mode number introduced in Eq. (52) and $B_{z}$ is replaced by $B_{\phi}$. The cylindrical slow continuum frequencies are given by $\omega_{s, m}(r)= \pm \bar{\omega}_{s}\left(n-\frac{m}{q(r)}\right)$ with from Eq. (73)

$$
\bar{\omega}_{s}(r)=\bar{\omega}_{A} \sqrt{\frac{\gamma p}{\gamma p+B^{2}}} \approx \bar{\omega}_{A} \sqrt{\frac{1}{2} \gamma \beta}=\frac{\sqrt{\gamma T}}{R_{0}} .
$$

The approximation of Eq. (75) holds for $\beta \equiv 2 p / B^{2} \ll 1$ and $B_{\theta} \| B_{Z}$. Both of the continuum frequencies vanish at the rational surface where $q=m / n$, indicating marginal stability in the absence of magnetic field line bending. It turns out that the only effect of poloidal flow $u_{\theta}$ and longitudinal flow $u_{z}$ on the cylindrical continuum frequencies is a Doppler shift to $\omega_{D} \equiv \omega+m u_{\theta} / r+k u_{z}$. 


\subsubsection{A torus}

In a torus, the poloidal angle is no longer an angle of symmetry. Whenever $n-m / q= \pm\left(n-m^{\prime} / q\right)$ so that $\omega_{A, m}=\omega_{A, m^{\prime}}$ and $\omega_{s, m}=\omega_{s, m^{\prime}}$, toroidal coupling between poloidal harmonics ensures that these cylindrical frequency crossings are avoided and a spectral gap appears. The gaps due to $\left|m-m^{\prime}\right|=1,2,3, \ldots$ are associated with poloidal variation of the equilibrium due to toroidicity, ellipticity, triangularity, etc.

In a torus there is also a coupling when $\omega_{A, m}=\omega_{s, m^{\prime}}$, which for $\beta \ll 1$ occurs approximately at the rational surface $q=m / n$ at a frequency

$$
\omega \approx\left(m-m^{\prime}\right) \bar{\omega}_{s} / q
$$

The continuous spectrum is substantially modified by all this coupling. As in a cylinder, rotation shifts the eigenfrequencies $\omega$ to a Doppler shifted frequency $\omega_{D} \equiv \omega+n \Omega$. Because in a torus the centrifugal force is no longer perpendicular to the magnetic surfaces, rotation introduces also more substantial changes to the continuous spectrum.

For a tokamak with isothermal magnetic surfaces, a small inverse aspect ratio $\epsilon \ll 1$, a Mach number of order unity, and low $\beta$ of order $\epsilon^{2}$, an expression for the low-frequency continuous Alfvén spectrum close to a rational surface was obtained analytically in Refs. [44,47]. Using the same assumptions, in Refs. $[45,46]$ the eigenfrequencies of axisymmetric $m=n=0$ modes were derived. According to Ref. [65], at the rational surface these frequencies are equal. ${ }^{1}$ Repeating the procedure of Refs. $[45,46]$ for arbitrary $\gamma_{\zeta}=\zeta /(\zeta-1)$, we derived ${ }^{2}$ the eigenfrequencies $\omega_{D}=0, \omega_{D}=\bar{\omega}_{s}^{2} / q^{2}$, and $\omega_{D}^{2}=\omega_{ \pm}^{2}$, where

$$
\omega_{ \pm}^{2}=\bar{\omega}_{s}^{2}\left(a_{1} \pm \sqrt{a_{1}^{2}-a_{2}}\right)
$$

and,

$$
\begin{aligned}
& a_{1}=1+\frac{1}{2 q^{2}}+2 M^{2}+\frac{\gamma}{\gamma_{\zeta}} \frac{M^{4}}{4}, \\
& a_{2}=\left(\frac{\gamma}{\gamma_{\zeta}}-1\right) \frac{M^{4}}{2 q^{2}},
\end{aligned}
$$

where $M=R_{0} \Omega / \sqrt{\gamma T}$ and $\bar{\omega}_{s}=\sqrt{\gamma T} / R_{0}$. In the limit $\zeta \rightarrow \infty\left(\gamma_{\zeta} \rightarrow 1\right)$ for isothermal magnetic surfaces, this result agrees with that of Refs. [44,65,47] for the low-frequency Alfvén continuum. In the limit $\zeta \rightarrow 1\left(\gamma_{\zeta} \rightarrow \infty\right)$ for magnetic surfaces of constant density the recent result of Refs. [48] for axisymmetric modes is recovered. The present result generalizes these particular results to arbitrary $\gamma_{\zeta}$.

For small flow velocities $M^{2} \ll 1$, we obtain from Eq. (77a)

$$
\omega_{-}^{2} \approx\left(\frac{\gamma}{\gamma_{\zeta}}-1\right) \frac{M^{2} \Omega^{2}}{2\left(1+2 q^{2}\right)},
$$

which is equal to the BV-frequency $\omega_{\mathrm{BV} \text {, tokamak }}^{2}$ of Eq. (51). This clearly shows that the flow-induced continuum gap for $\gamma>\gamma_{\zeta}$ between $\omega_{D}=-\omega_{-}$and $\omega_{-}$can be attributed to the convective effect, as was suggested in Refs. [66,44].

In the absence of flow, the frequencies of Eq. (77a) are given by $\omega_{-}^{2}=0$ and $\omega_{+}^{2}=\left(1+2 q^{2}\right) \bar{\omega}_{s} / 2 q^{2}$. The so-called beta-induced continuum gap between $\omega_{D}=-\omega_{+}$and $\omega_{+}$was first found in Refs. [67,68]. Note the similarity with the slow-Alfvén coupling frequency Eq. (76), the difference being half the Pfirsch-Schlüter factor $1+2 q^{2}$. This toroidal effect influences the continuum modes through the curvature of the magnetic field within the magnetic surfaces, called the geodesic curvature. These $\omega_{+}$-continuum modes are therefore often referred to as Geodesic Acoustic Modes (GAMs) [69].

We note that for $M=0$ the zero-frequency continuum modes $\omega_{-}=0$ can be thought of as equilibrium flows described by Eq. (16). These flows are the linear counterpart of zonal flows. We finally note that, away from the resonant surface, the coefficients $a_{1}$ and $a_{2}$ acquire additional terms $(m / q-n)^{2} / \gamma \beta$ and $2(m / q-n)^{2} / \gamma \beta q^{2}$, respectively, to account for the effect of magnetic field line bending [44].

\section{Numerical calculations}

In this section we numerically analyse the waves and instabilities arising in the analytical equilibria of Eq. (21). The discrete spectrum of global modes and instabilities arising in these equilibria have been discussed in Refs. [70] and [64], respectively. Here, we will focus on the low-frequency continuous MHD spectrum. In particular we will look at the continuum

\footnotetext{
${ }^{1}$ The result of Refs. [45,46] for axisymmetric modes however differs from that of Refs. [44,47] for general mode number continuum modes. This can be attributed to an erroneous factor of -2 in the exponential dependence of the density of Ref. [45], of which only the minus sign was corrected in Ref. [46]. In addition, a factor 2 is missing in the second term in Eq. (15) of Ref. [46].

${ }^{2}$ The difference in the derivation of Eq. (77a) compared to the result of Refs. [45,46] for $\gamma_{\zeta} \rightarrow 1$ and [48] for $\gamma_{\zeta} \rightarrow \infty$, pertains to evaluation of the advection of $\rho$ and $S$ by the perturbed velocity $\mathbf{v}$ lying within the magnetic surfaces. Using the general parametrization of Eqs. (10) and (11), for general $\gamma_{\zeta}$ one finds $\mathbf{v} \cdot \nabla S=\left(\gamma_{\zeta}-\gamma\right)(S / \rho) \mathbf{v} \cdot \nabla \rho$ and $\mathbf{v} \cdot \nabla \rho=v_{R} \rho R \Omega^{2} \mid \gamma_{\zeta} T$.
} 
frequencies $\omega_{D}^{2}=\omega_{-}^{2}$ given by Eq. (77a). We consider the cases $\zeta=\gamma /(\gamma-1), \zeta \rightarrow \infty$, and $\zeta \rightarrow 1$ corresponding to isentropic $(S(\psi))$, isothermal $(T(\psi))$, and isochoric $(\rho(\psi))$ magnetic surfaces.

\subsection{Solvers}

To obtain a numerical representation of the analytical equilibria in the coordinates required for the stability analysis, the equilibrium code FINESSE [39] is used. This FINite Element Solver for Stationary Equilibria, can calculate equilibria with general equilibrium flows in several geometries. In case of purely toroidal flow, one can choose a constant $\gamma_{\zeta}$ so that the quantity $A(\psi)=p \rho^{-\gamma_{\zeta}}$ from Eq. (14) is a flux function. The limits $\gamma_{\zeta} \rightarrow \infty$ and $\gamma_{\zeta} \rightarrow 1$, for isochoric and isothermal flux surfaces respectively, are included as special cases [40]. Other dedicated equilibrium codes that include both toroidal and poloidal flow are CLIO [26] and FLOW [71]. FINESSE uses a Galerkin approach with isoparametric bicubic Hermite basis functions for quartic convergence behavior.

The linear stability code PHOENIX [72] is used to numerically investigate the continuous spectra. This mixed spectral and finite element code was developed from the CASTOR code [28] by including the ability to treat arbitrary equilibrium flow. The linearised MHD equations result in a generalized eigenvalue problem that is efficiently solved in PHOENIX using the iterative Jacobi-Davidson algorithm [73]. The singular continuum eigenfunctions, obviously, cannot be resolved by the continuous finite element basis functions. The associated eigenfrequencies however can be accurately found by replacing the basis functions by well-ordered constants [74].

\subsection{Equilibria}

The equilibria considered in this section are those of Eq. (21). The coefficients $c_{1}$ and $c_{2}$ are adjusted using Eqs. (27) and (29) to give an inverse aspect ratio $\epsilon=0.1$ and ellipticity $E=1$. The constant $\Lambda=\Omega^{2} / 2 T_{s}$ from Eq. (19) is adjusted to yield a desired central Mach number $M_{0} \equiv M\left(R=R_{0}\right)$. The ratio $P / J$ is used to give a $\beta$-profile ranging from zero at the plasma edge to a maximum of approximately 0.005 . The constant $J$ is obtained as an eigenvalue by the solver so that a normalized flux function $\psi$ between zero at the magnetic axis and one at the edge is obtained [39]. The overall magnitude of $\psi$ was used to rescale the safety factor $q$. The resulting relatively flat $q$-profile was centred around $q=2$ positioned at $s \approx 0.68$, where

$$
s \equiv \sqrt{\frac{\psi-\psi_{m}}{\psi_{e}-\psi_{m}}}
$$

with $\psi_{e}$ and $\psi_{m}$ the values of $\psi$ at the edge and the magnetic axis, respectively. A ratio of specific heats $\gamma=5 / 3$ was used in all the calculations. The parametric dependence of $\Omega, T_{s}$, and $\rho_{s}$ is restrained by the ideal gas law $p_{s}=\rho_{s} T_{s}$ and the assumption that $\Lambda$ is constant. First, the remaining freedom is used to investigate the effect of rigid rotation, for which $T_{s}$ is constant. In Section 6.4.3, $\Omega^{2}, T_{s}$, and $p_{s}$ are assumed to be of the same form so that $\rho_{s}$ is constant.

\subsection{Convergence}

For equilibria including toroidal flow, the analytical solutions of Eq. (21) are ideally suited to test the accuracy and convergence behavior of a numerical solver. In this section we show the results of such a test for the equilibrium code FINESSE [39]. The equilibria described in the previous section were used with isochoric flux surfaces $\zeta=1$, so that the analytical expression Eq. (39) for the location of the magnetic axis could be used. This allowed the analytical solution to be compared

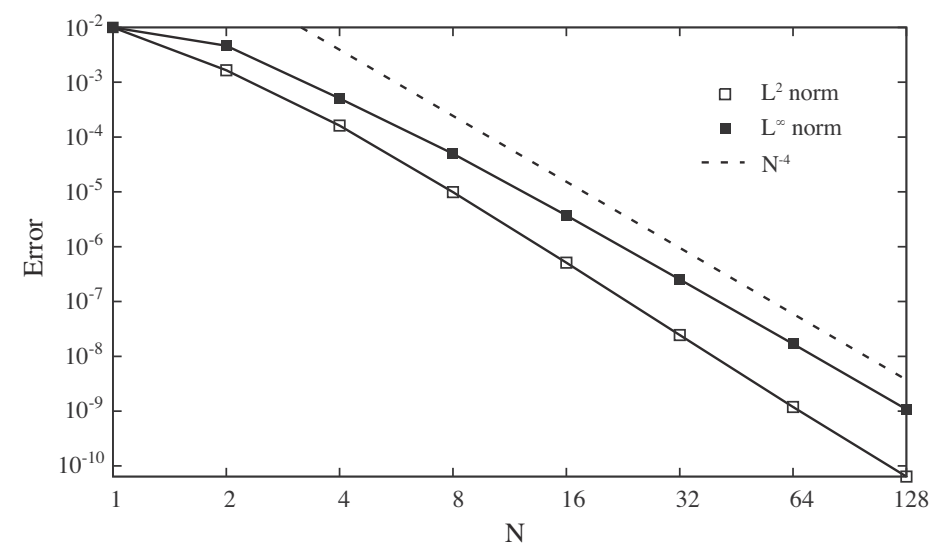

Fig. 2. The $L^{n}$-norm difference $\left\|\psi_{\text {num. }}-\psi_{\text {an. }}\right\|_{n}$ between the numerical solution $\psi_{\text {num. }}$ and the analytical solution $\psi_{\text {an. }}$ of Eq. (21) with $\zeta=1$ as a function of the number $N$ of finite elements in the radial and the poloidal direction. 
with the numerical solution, which is scaled between zero on the magnetic axis and one at the plasma edge. A central Mach number $M_{0}=1$ was used.

In Fig. 2 the difference between the analytical and the numerical solution is shown in both the $L^{2}$ and the $L^{\infty}$ norm, i.e. the root mean square of the difference and the maximum difference. Both measures of the error go down with the number $N$ of radial and poloidal elements approximately as $1 / N^{4}$, demonstrating fourth order convergence up to a very small error of less than $10^{-10}$.

\subsection{Continuous spectra}

In this section, we will investigate the solutions of Eq. (21) for $\zeta=1, \zeta \rightarrow \infty$, and $\zeta=\gamma /(\gamma-1)$, resulting in magnetic surfaces with constant density, temperature, and entropy, respectively.

\subsubsection{Isentropic magnetic surfaces}

Fig. 3 shows part of the continuous spectrum for the case that the entropy is constant on the magnetic surfaces. As discussed in Section 2.3.1, this may be appropriate in the presence of significant poloidal flow. The frequencies are Doppler shifted and nondimensionalized with $\bar{\omega}_{A m}$, which is $\bar{\omega}_{A}$ evaluated at the magnetic axis. The continuum frequencies are displayed as a function of the radial coordinate $s$ of Eq. (79), which vanishes at the magnetic axis and assumes the value $s=1$ at the plasma edge.

Two different continuum branches are shown, corresponding to the cylindrical continua of Section 5.6.2. The lowest frequency branch corresponds to slow magnetosonic waves, while the higher frequency branch corresponds to Alfvén waves. As elaborated in Section 5.6.3, compressibility and toroidicity allow Alfvén waves to couple with the slow magnetosonic waves so that the continua acquire a mixed slow-Alfvén polarization. Some cylindrical characteristics however remain, such as the diverging frequency of the Alfvén-like branch towards the plasma edge where the density vanishes.

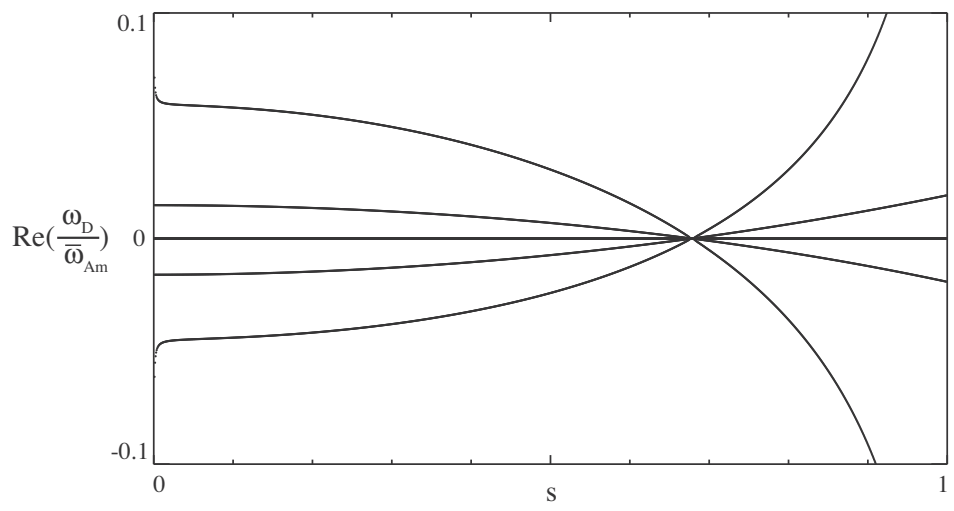

Fig. 3. The Doppler shifted frequency $\omega_{D}$, normalized by $\omega_{A m} \equiv \bar{\omega}_{A}\left(\psi_{m}\right)$, as a function of the radial coordinate $s$. The frequencies of the shown $n=1$ continuum branches vanish at the rational surface at $s \approx 0.68$. The analytical equilibrium solution with isentropic flux surfaces $(\zeta=\gamma /(\gamma-1))$ is used for $M_{0}=1$.

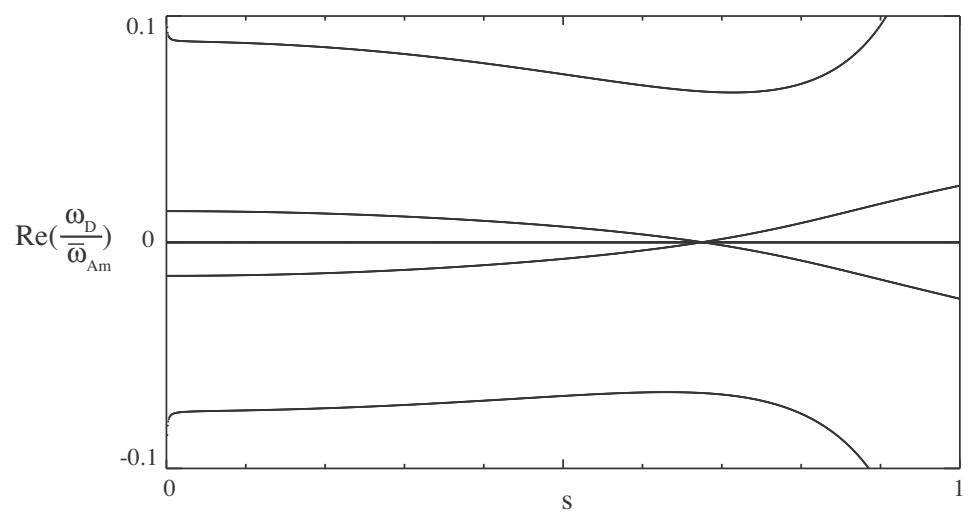

Fig. 4. The low-frequency $n=1$ continuous spectrum of the analytical equilibrium with isothermal flux surfaces $(\zeta \rightarrow \infty)$ for $M_{0}=1$, showing the appearance of a flow-induced continuum gap due to the convective effect. 
A toroidal mode number $n=1$ was used so that in a cylinder, according to Section 5.6 .2 , the frequency of modes with a poloidal mode number $m=2$ vanish at the rational surface $q=m / n=2$. Close to the rational surface, the Alfvén-like frequency branch is described by $\omega_{D}= \pm \omega_{-}$from Eq. (77a). In a toroidal plasma with isentropic flux surfaces $\gamma=\gamma_{\zeta}$ so that $a_{2}$ of Eq. (77c) vanishes. According to Eq. (77a), $\omega_{-}$vanishes in this case at the rational surface as well. As explained in Section 4.2 .2 , this is due to the fact that without entropy gradients the convective effect is absent.

\subsubsection{Isothermal magnetic surfaces}

Fig. 4 shows the same part of the spectrum as Fig. 3, but now the limit $\zeta \rightarrow \infty$ is taken in the equilibrium solution (21) so that the magnetic surfaces become isothermal. The lowest frequency branch is only slightly affected, while the higher frequency branch is lifted upwards. These continuum frequencies are described quite accurately by $\omega_{D}= \pm \omega_{-}$from Eq. (77a).

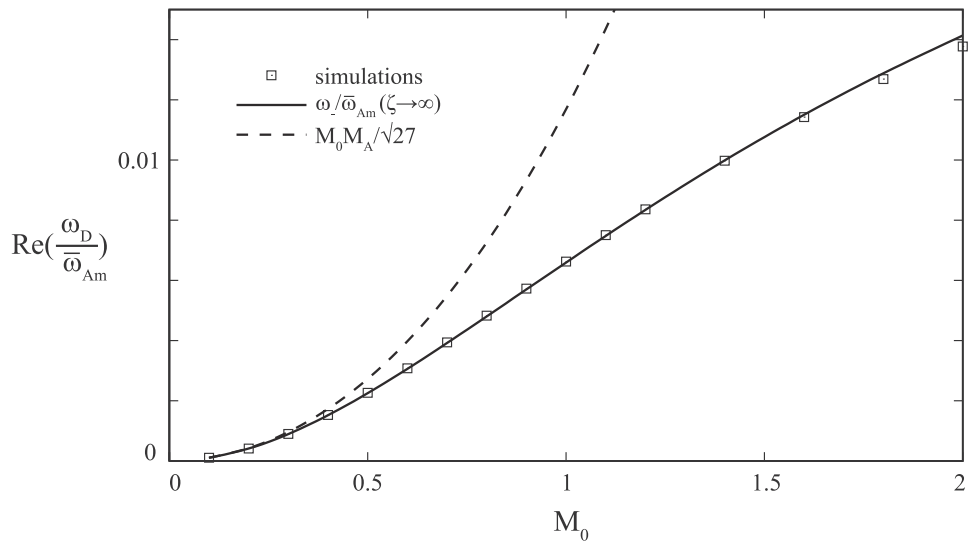

Fig. 5. The half-width of the flow-induced continuum gap of the analytical equilibrium with isothermal magnetic surfaces ( $\zeta \rightarrow \infty$ ) as a function of the Mach number $M_{0}$. The numerical values (boxes) are compared with the analytical expression for $\omega_{-}$from Eq. (77a) (solid line) and its approximation Eq. (78) for low $M_{0}$ (dashed line) for $q=2$. Here $M_{A}=\Omega / \bar{\omega}_{A m}$ is the Alfvén Mach number.

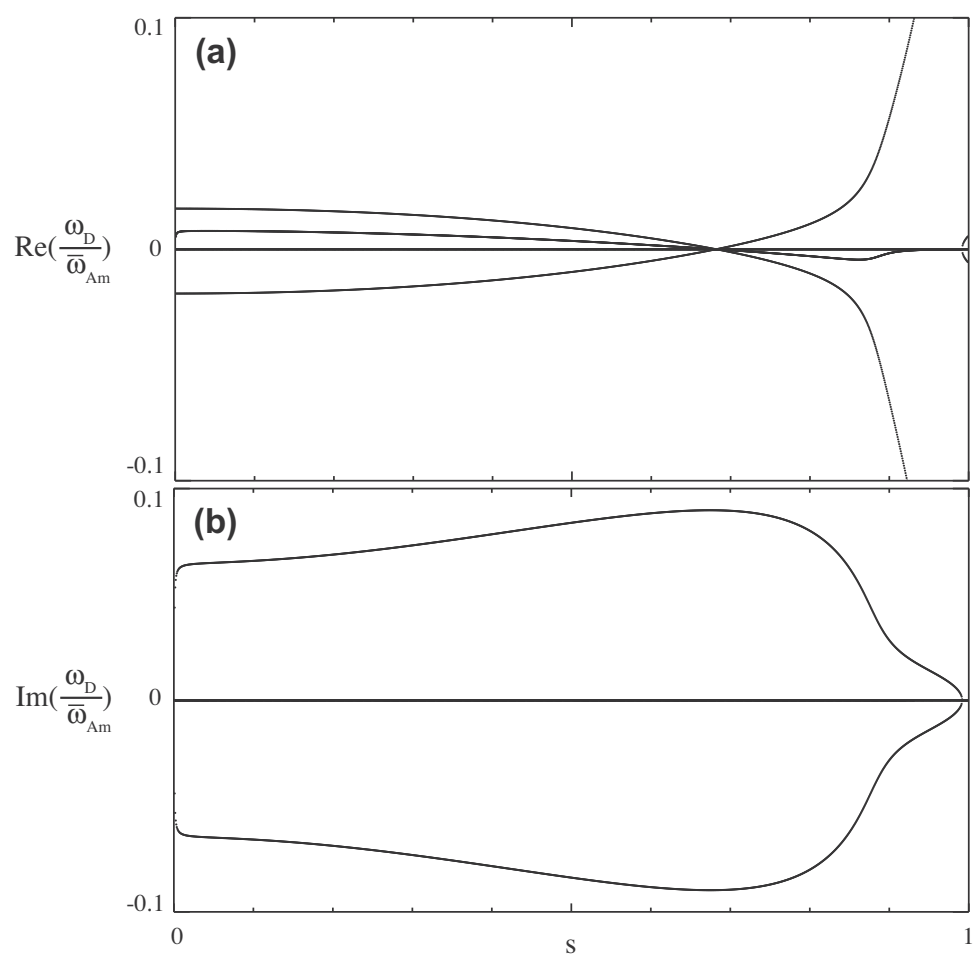

Fig. 6. The real (a) and imaginary (b) part of the low-frequency $n=1$ continuous spectrum of the analytical equilibrium with isochoric flux surfaces $(\zeta=1$ ) for $M_{0}=1$. Two of the four continuum branches that are stable near the plasma edge at $s=1$ form a complex conjugate pair of unstable eigenmodes by entering the complex plane. Their common real frequency deviates slightly from the Doppler shift due to the Coriolis effect. 
Rotation lifts these continuum frequencies to a finite buoyancy frequency or BV frequency due to the negative entropy gradients in the direction of the centrifugal force within the isothermal magnetic surfaces [44].

Fig. 5 shows the half-width of the flow-induced continuum gap obtained from the numerical computations, as a function of the Mach number $M_{0} \equiv M\left(R_{0}\right)$. These numerical results are compared with the analytical result for $\omega_{-}$of Eq. (77a) and the approximation of Eq. (78), for low Mach numbers. Surprisingly good correspondence with $\omega_{-}$is found up to sonic Mach numbers, even though the aspect ratio $\epsilon=0.1$ only just satisfies the assumption $\epsilon \ll 1$ used in the derivation of this analytical result. For higher Mach numbers, the flux surfaces start to acquire significant triangularity and the computational results start to deviate slightly from the analytical expression. Due to the excellent correspondence, a comparison such as performed here provides a good standardized test for stability codes including toroidal rotation.

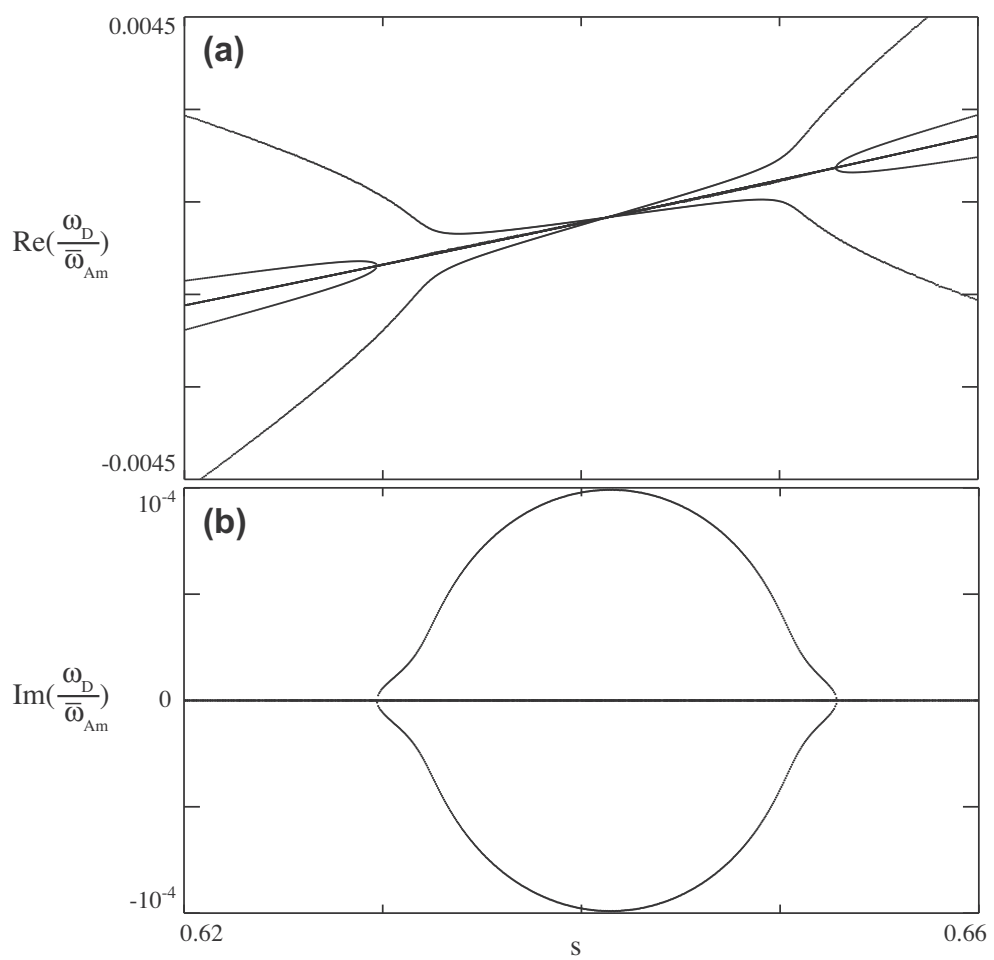

Fig. 7. The real (a) and imaginary (b) part of the $n=1$ low-frequency spectrum of the analytical equilibrium with constant density. For $M_{0}=0.1$ only a small part of the tokamak is unstable (note the abscissa).

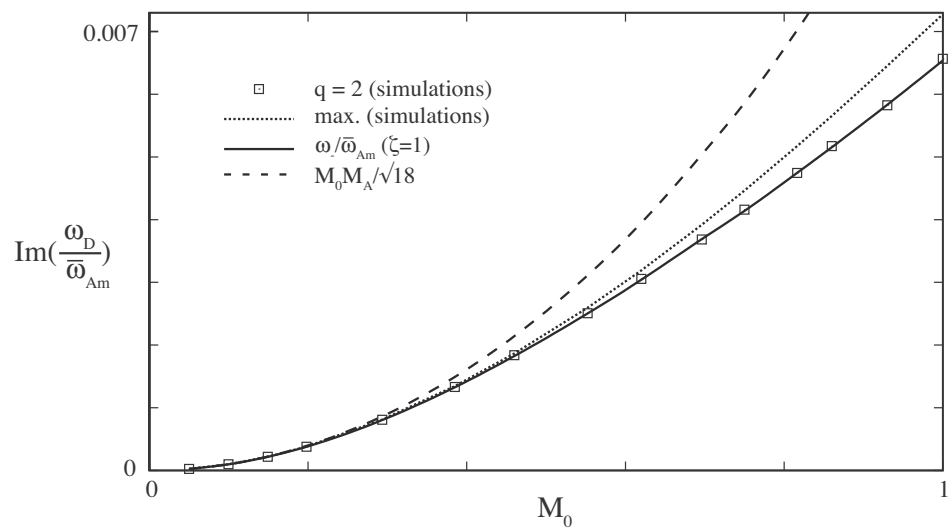

Fig. 8. The growth rate of the unstable continuous spectrum of the constant density analytical equilibrium as a function of the Mach number $M_{0}$. The numerical values for the growth rate at the rational $q=2$ surface (boxes) and the maximum growth rate (dotted line) are compared with the analytical expression for $\operatorname{Im}\left(\omega_{-}\right)$of Eq. (77a) (solid line) and the approximation Eq. (78) for low $M_{0}$ (dashed line), for $q=2$. Here $M_{A} \equiv \Omega / \bar{\omega}_{A m}$ is evaluated at the rational surface. 


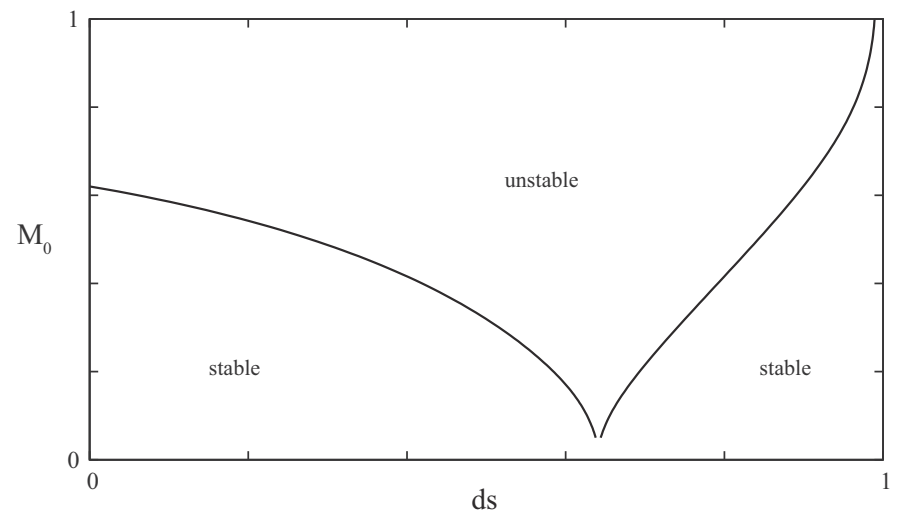

Fig. 9. The radial extent over which the constant density analytical equilibrium is unstable, as a function of the Mach number $M_{0}$. For low $M_{0}$, only a small region near the rational surface is unstable, to spread to almost the entire tokamak for $M_{0}=1$.

\subsubsection{Isochoric magnetic surfaces}

Fig. 6(a) shows again the same part of the spectrum as Figs. 3 and 4, but now for the equilibrium of Eq. (21) with $\zeta=1$ so that the magnetic surfaces have constant density. The low-frequency slow-like branch seems similar to that of Figs. 3 and 4 . Past the rational surface however, the frequency of this continuum diverges as is expected of Alfvén-like waves. The branch that previously was Alfvén-like, now has actually become lower in frequency than the continuum branch that was slow-like. Apart from a very small region near the plasma edge, these continua even leave the real axis of the complex frequency plane by acquiring an imaginary component.

The imaginary component of the frequency is shown in Fig. 6(b). As implied by Eq. (60), the unstable continuum modes turn up as a complex conjugate pair. By Eq. (52), the continuum mode with a positive imaginary part of the frequency is unstable, while the mode with a negative imaginary part is damped. Because these modes also have a real frequency, these modes oscillate while their amplitude increases (over-stable) or decreases. Note from Fig. 6(a) that the real part of the Doppler shifted frequency of this complex conjugate pair of modes only slightly deviates from zero, corresponding to the small Coriolis shift $\bar{V}$ of Eq. (60). Note from Fig. 6 that the highest growth rate of the continuum modes is close to the rational surface at $s \approx 0.68$, where the magnetic field line bending vanishes. The growth rate decreases towards the plasma edge, primarily due to the effect of the decreasing density on the remaining Alfvén-like character of the modes.

Finally, we investigate the maximum growth rate of the continuous spectrum as a function of the Mach number $M_{0}$. We do this for a tokamak with a constant static density $\rho_{s}$ over the entire plasma. In order to satisfy the assumptions of Eq. (19), this implies that the angular frequency squared goes down towards the plasma edge like the static pressure $p_{s}$. Fig. 7(a) shows for $M_{0}=0.1$ the continuum frequencies in a very small region around the rational surface $q=2$. Because the angular frequency varies over the tokamak, the Doppler shift is no longer constant and the actual frequency $\omega$ is shown. The Alfvénlike continuum again becomes unstable over some part of the plasma, resulting in the imaginary part of the frequency shown in Fig. 7(b). When the Mach number is increased, both the maximum growth rate and the radial extent over which the continuous spectrum is unstable increases. This is shown in Figs. 8 and 9, respectively. From Fig. 9, the region over which the tokamak becomes unstable spreads with increasing $M_{0}$ from a tiny region close to the rational surface, to almost the entire tokamak for $M_{0}=1$.

In Fig. 8 the growth rate at the rational surface obtained numerically is compared to $\operatorname{Im}\left(\omega_{-}\right)$of Eq. (77a) and the approximation of Eq. (78). Note that for constant $\Lambda$, in the large aspect ratio limit the Mach number $M$ of Eq. (77a), evaluated at the rational surface, is equal to $M_{0}$. Also shown is the maximum growth rate of the continuum, which occurs closer to the magnetic axis, where the angular frequency is higher. Excellent agreement is obtained between the numerically obtained growth rate at the rational surface and the analytical result, showing the value of such a comparison for the validation of numerical stability codes.

\section{Conclusion}

The axisymmetric stationary ideal MHD equations are reduced to an extended Grad-Shafranov equation Eq. (18) that allows one to specify which thermodynamic quantity is constant on the magnetic surfaces. Analytical equilibrium solutions (21) have been derived that still contain this freedom. An arbitrary number of vacuum solutions may be added, allowing control over characteristics like the aspect ratio, ellipticity, and triangularity of the equilibrium. Expressions for the Mach number, average beta, and safety factor are derived, revealing some restrictions on attainable values.

The analytical solutions have been used to show the fourth order convergence of a numerical code, showing their usefulness for testing numerical equilibrium codes including toroidal rotation. 
The frequencies of the continuous Alfvén spectrum close to a rational surface have been derived including the effects of toroidicity, compressibility, and rotation. These expressions (77a) still possess the freedom to specify which thermodynamic quantity of the underlying equilibrium is constant on the magnetic surfaces.

The lowest Alfvén continuum frequency of a slowly rotating tokamak is shown to be equal to a heuristically derived expression, Eq. (51), for the Brunt-Väisälä-frequency due to the centrifugal convective effect on plasma confined to the magnetic surfaces.

Depending on which quantity is constant on the magnetic surfaces, the convective effect either lifts the continuum frequencies to a finite value or destabilizes the continuum. The analytical expressions for the flow-induced gap and the growth rate of the unstable continuum show excellent agreement with the numerical results, showing their merits for the validation of stability codes including toroidal rotation.

The present analytical spectral and equilibrium results generalize various previous results in which initial assumptions were made on which thermodynamic quantity is constant on magnetic surfaces. This work shows that in many cases no such assumption needs to be made at forehand. It is therefore recommended that, when considering purely toroidal rotation, the general parametrizations of Eqs. (10) and (11) for the pressure and the density are used to make subsequent analysis as general as possible. The same holds for numerical codes including purely toroidal rotation, where typically the specific choice of isothermal magnetic surfaces is made, unnecessarily restricting the generality offered by the MHD equations.

\section{Acknowledgments}

We gratefully acknowledge the valuable comments received from J. W. S. Blokland on a draft version of the manuscript. This work, supported by NWO and the European Communities under the contract of the Association EURATOM/FOM, was carried out within the framework of the European Fusion Program. The views and opinions expressed herein do not necessarily reflect those of the European Commission.

\section{References}

[1] H. Grad, H. Rubin, Proceedings of the Second United Nations Conference on the Peaceful Uses of Atomic Energy, vol. 21, 1958, pp. 190-197.

[2] V.D. Shafranov, On magnetohydrodynamic equilibrium configurations, Soviet Phys. JETP 33 (1957) 710-722.

[3] L.S. Solov'ev, The theory of hydromagnetic stability of toroidal plasma configurations, Soviet Phys. JETP 26 (1968) $400-407$.

[4] M.J.M. Hill, On a spherical vortex, Phyl. Trans. Roy. Soc. London A 185 (1894) 213-245.

[5] E.K. Maschke, Exact solutions of the MHD equilibrium equation for a toroidal plasma, Plasma Phys. 15 (1973) 535-541.

[6] F. Herrnegger, On the equilibrium and stability of the belt pinch, in: Proceedings 5th European Conference on Controlled Fusion Plasma Physics, 1972, p. 26.

[7] L. Guazzotto, J.P. Freidberg, A family of analytic equilibrium solutions for the Grad-Shafranov equation, Phys. Plasmas 14 (2007) 112508.

[8] J.P. Sudano, Equilibrium of a toroidal plasma, Phys. Fluids 17 (1974) 1915-1916.

[9] C.S. Lai, M. Verleun, An exact solution for toroidal plasma in equilibrium, Phys. Fluids 19 (1976) 1066-1067.

[10] P.J. Mc Carthy, Analytical solutions to the Grad-Shafranov equation for tokamak equilibrium with dissimilar source functions, J. Plasma Fusion Res. 6 (1999) 937-948.

[11] S. Wang, J. Yu, An exact solution of the Grad-Shafranov-Helmhotz equation with central current density reversal, Phys. Plasmas 12 (2005) 062501.

[12] J. Scheffel, Linear MHD equilibria in toroidal geometry-exact and approximate solutions, Phys. Scr. 30 (1984) $350-365$.

[13] C.V. Atanasiu, S. Günter, K. Lackner, I.G. Miron, Analytical solutions to the Grad-Shafranov equation, Phys. Plasmas 11 (2004) $3510-3518$.

[14] K.H. Tsui, Toroidal equilibria in spherical coordinates, Phys. Plasmas 15 (2008) 112506.

[15] A.H. Khater, S.M. Moawad, Exact solutions for axisymmetric nonlinear magnetohydrodynamic equilibria of aligned magnetic field and plasma flow with applications to astrophysics and plasma confinement devices, Phys. Plasmas 16 (2009) 052504.

[16] S.B. Zheng, A.J. Wootton, E.R. Solano, Analytical tokamak equilibrium for shaped plasmas, Phys. Plasmas 3 (1996) $1176-1178$.

[17] A.J. Cerfon, J.P. Freidberg, One size fits all analytic solutions to the Grad-Shafranov equation, Phys. Plasmas 17 (2010) 032502.

[18] E.K. Maschke, H. Perrin, Exact analytic solution of the stationary M.H.D. equilibrium equation of a toroidal plasma in rotation, Plasma Phys. 22 (1980) 579-594.

[19] G.N. Throumoulopoulos, G.N. Pantis, Analytic axisymmetric magnetohydrodynamic equilibria of a plasma torus with toroidal mass flow, Phys. Fluids B 1 (1989) 1827-1833.

[20] R.A. Clemente, R. Farengo, A class of rotating compact tori equilibria, Phys. Fluids 27 (1984) $776-778$.

[21] O. Missiato, J.P. Sudano, Exact analytic solution of the stationary M.H.D. equilibrium equation of a toroidal plasma in rotation, in: Conference: LatinAmerican workshop on plasma physics and controlled nuclear fusion research, Cambuquira, Brazil, 8 February 1982, Rev. Bras. Fis. J. 1 (1982) $264-275$.

[22] R.L. Viana, R.A. Clemente, S.R. Lopes, Spherically symmetric stationary MHD equilibria with azimuthal rotation, Plasma Phys. Control Fusion 39 (1997) 415-434.

[23] G.N. Throumoulopoulos, G. Poulipoulis, G. Pantis, H. Tasso, Exact magnetohydrodynamic equilibria with flow and effects on the Shafranov shift, Phys. Lett. A 317 (2003) 463-469.

[24] H. Tasso, G.N. Throumoulopoulos, Axisymmetric ideal magnetohydrodynamic equilibria with incompressible flows, Phys. Plasmas 5 (1998) 23782383.

[25] J.P. Goedbloed, Some remarks on computing axisymmetric equilibria, Comput. Phys. Commun. 31 (1984) $123-135$.

[26] S. Semenzato, R. Gruber, H.P. Zehrfeld, Computation of symmetric ideal MHD flow equilibria, Comput. Phys. Rep. 1 (1984) 389-425.

[27] H. Lütjens, A. Bondeson, A. Roy, Axisymmetric MHD equilibrium solver with bicubic Hermite elements, Comput. Phys. Commun. 69 (1992) 287-298.

[28] W. Kerner, J.P. Goedbloed, G.T.A. Huysmans, S. Poedts, E. Schwarz, CASTOR: Normal-mode analysis of resistive MHD plasmas, J. Comput. Phys. 142 (1998) 271-303.

[29] R. Keppens, J.W.S. Blokland, Computing ideal magnetohydrodynamic equilibria, Trans. Fusion Sci. Technol. 49 (2006) $131-138$.

[30] W. Kerner, O. Jandl, Axisymmetric MHD equilibria with flow, Comput. Phys. Commun. 31 (1984) $269-285$.

[31] L.L. Lao, Variational moment method for computing magnetohydrodynamic equilibria, Comput. Phys. Commun. 31 (1984) 201-212.

[32] W. Kerner, S. Tokuda, Computation of tokamak equilibria with steady flow, Zeitschr. Naturforsch. A 42 (1987) 1154-1166.

[33] W.A. Cooper, S.P. Hirshman, Axisymmetric MHD equilibria with isothermal toroidal mass flow by variational steepest descent method, Plasma Phys. Controlled Fusion 29 (1987) 933-943. 
[34] V. Varadarajan, W.H. Choe, G.H. Miley, Rapid calculation of tokamak equilibria with toroidal rotation via minimization of a functional, Appl. Math. Comput. 43 (1991) 145-163.

[35] F. Masaru, N. Yuji, H. Satoshi, W. Masahiro, Tokamak equilibria with toroidal flows, J. Plasma Fusion Res. 76 (2000) $937-948$.

[36] R. Qilong, Z. Cheng, Numerical study of tokamak equilibrium with toroidal flow on EAST, Plasma Sci. Technol. 8 (2006) $511-515$.

[37] K. Elsasser, A. Heimsoth, Axisymmetric plasma equilibria with incompressible flow, Zeitschr. Naturforsch. A 41 (1986) $883-896$.

[38] V.D. Varadarajan, G.H. Miley, Linear instability analysis for toroidal plasma flow equilibria, J. Comput. Phys. 123 (1996) $415-434$.

[39] A.J.C. Beliën, M.A. Botchev, J.P. Goedbloed, B. van der Holst, R. Keppens, FINESSE: Axisymmetric MHD equilibria with flow, J. Comput. Phys. 11 (2002) 91-117.

[40] J.W.S. Blokland, R. Keppens, J.P. Goedbloed, Unstable magnetohydrodynamical continuous spectrum of accretion disks, Astronomy Astrophys. 467 (2007) 21-35.

[41] D. Berger, L.C. Bernard, R. Gruber, F. Troyon, Numerical study of the unstable MHD spectrum of a small aspect ratio, flat current, non-circular tokamak, J. Appl. Math. Phys. 31 (1980) 113-132.

[42] R.C. Grimm, R.L. Dewar, J. Manickam, Ideal MHD stability calculations in axisymmetric toroidal coordinate systems, J. Comput. Phys. 49 (1983) 94-117.

[43] A.R. Schellhase, R.G. Storer, Spectral analysis of resistive MHD in toroidal geometry, J. Comput. Phys. 123 (1996) 15-31.

[44] B. van der Holst, A.J.C. Beliën, J.P. Goedbloed, Low frequency Alfvén waves induced by toroidal flows, Phys. Plasmas 7 (2000) $4208-4222$.

[45] S. Wang, Zonal flows in tokamak plasmas with toroidal rotation, Phys. Rev. Lett. 97 (2006) 085002.

[46] S. Wang, Erratum: Zonal flows in tokamak plasmas with tororidal rotation, Phys. Rev. Lett. 97 (2006) 129902.

[47] C. Wahlberg, Low-frequency magnetohydrodynamics and geodesic acoustic modes in toroidally rotating tokamak plasmas, Plasma Phys. Control Fusion 51 (2009) 085006.

[48] V.P. Lakhin, V.I. Ilgisonis, A.I. Smolyakov, Geodesic acoustic modes and zonal flows in toroidally rotating tokamak plasmas, Phys. Lett. A 374 (2010) 4872-4875.

[49] E. Hameiri, The equilibrium and stability of rotating plasmas, Phys. Fluids 26 (1983) 230-237.

[50] H.P. Zehrfeld, B.J. Green, Stationary toroidal equilibria at finite beta, Nucl. Fusion 12 (1972) 569-575

[51] K. Avinash, S.N. Bhattacharyya, B.J. Green, Axisymmetric toroidal equilibrium with incompressible flows, Plasma Phys. Controlled Fusion 11 (1992) $465-473$.

[52] C. Yarim, U. Daybelge, A. Nicolai, Modelling of Rotating Plasma States and their Stability Accounting for Neutral Beam Injection and Helical Perturbations, Technical Report, Juelich Research Center, 2003.

[53] E.P. Velikhov, Stability of an ideally conducting liquid flowing between cylinders rotating in a magnetic field, Sov. Phys. JETP 36 (1959) $1398-1404$.

[54] S. Chandrasekhar, The stability of non-dissipative couette flow in hydromagnetics, Proc. Nat. Acad. Sci. 46 (1960) $253-257$.

[55] S.A. Balbus, J.F. Hawley, A powerful local shear instability in weakly magnetized disks. I: Linear analysis, Astrophys. J. 376 (1991) $214-222$.

[56] M. Furukawa, Z. Yoshida, M. Hirota, V. Krishan, MHD stability in flowing plasmas: Connection between fusion plasma and astrophysics research, Plasma Fusion Res. 2 (2007) 016

[57] I.B. Bernstein, E.A. Frieman, M.D. Kruskal, R.M. Kulsrud, An energy principle for hydromagnetic stability problems, Proc. Roy. Soc. London 244 (1958) 17-40.

[58] E. Frieman, M. Rotenberg, On hydromagnetic stability of stationary equilibria, Rev. Mod. Phys. 32 (1960) 898-902

[59] F.L. Waelbroeck, Gyroscopic stabilization of the internal kink mode, Phys. Plasmas 3 (1996) 1047-1053.

[60] J.P. Goedbloed, New construction of the magnetohydrodynamic spectrum of stationary plasma flows. I: Solution path and alternator, Phys. Plasmas 16 (2009) 122110

[61] J.P. Goedbloed, S. Poedts, Principles of Magnetohydrodynamics, Cambridge University Press, 2004

[62] J.W.S. Blokland, E. van der Swaluw, R. Keppens, J.P. Goedbloed, Magneto-rotational overstability in accretion disks, Astronomy Astrophys. 444 (2005) 337-346.

[63] A.K. Sen, Stabilization of interchange type instabilities in tokamaks via inverted flow profiles, Nucl. Fusion 34 (1994) $459-463$.

[64] J.W. Haverkort, H.J. de Blank, Local stability of rotating tokamak plasmas, Plasma Phys. Control. Fusion 53 (2011) 045008.

[65] C. Wahlberg, Geodesic acoustic mode induced by toroidal rotation in tokamaks, Phys. Rev. Lett. 101 (2008) 115003.

[66] B.v.d. Holst, A.J.C. Beliën, J.P. Goedbloed, New Alfvén continuum gaps and global modes induced by toroidal flow, Phys. Rev. Lett. 84 (2000) $2865-2868$.

[67] M.S. Chu et al, A numerical study of the high-n shear Alfvén spectrum gap and the high-n gap mode, Phys. Fluids B 4 (1992) 3713-3721.

[68] A.D. Turnbull et al, Global Alfvén modes: Theory and experiment, Phys. Fluids B 5 (1993) 2546-2553.

[69] N. Winsor, J.L. Johnson, J.M. Dawson, Geodesic acoustic waves in hydromagnetic systems, Phys. Fluids 11 (1968) $2448-2450$.

[70] J.W. Haverkort, H.J. de Blank, B. Koren, Low-frequency Alfvén gap modes in rotating tokamak plasmas, Plasma Phys. Control Fusion 53 (2011) 045004.

[71] L. Guazzotto, R. Betti, J. Manickam, S. Kaye, Numerical study of tokamak equilibria with arbitrary flow, Phys. Plasmas 11 (2004) 604-614.

[72] J.W.S. Blokland, B. van der Holst, R. Keppens, J.P. Goedbloed, PHOENIX: MHD spectral code for rotating laboratory and gravitating astrophysical plasmas, J. Comput. Phys. 206 (2007) 509-533.

[73] G.L.G. Sleijpen, H.A. van der Vorst, A Jacobi-Davidson iteration method for linear eigenvalue problems, SIAM J. Matrix Anal. Appl. 17 (1996) $401-425$.

[74] S. Poedts, E. Schwarz, Computation of the ideal-MHD continuous spectrum in axisymmetric plasmas, J. Comput. Phys. 105 (1993) $165-168$. 